\title{
Calculation of fluid-structure interaction: methods, refinements, applications
}

\author{
M. Can Kara MSc, PhD \\ Engineer, Det Norske Veritas (DNV) USA Inc., Houston, TX, USA \\ T. Stoesser PhD, Dipl-Ing. \\ Professor, School of Engineering, Cardiff University, Cardiff, UK
}

R. McSherry PhD

Research Associate, School of Engineering, Cardiff University, Cardiff, UK

This paper starts by reviewing the most significant previous studies on fluid-structure interaction of flows past stationary and moving circular cylinders, including those undergoing fluid dynamic excitation in the form of vortexinduced vibrations. It shows the importance of flow past a circular cylinder as a convenient validation test case for new numerical methods and demonstrates the advantages of the immersed boundary method. A new method based on the immersed boundary method to accurately compute fluid-structure interaction and vortex-induced vibrations is then presented and applied. The fluid solver is strongly coupled with the equation of motion through Hamming's fourth-order predictor-corrector method, which allows computation of the interaction of a fluid with a moving structure. The accuracy, validity and efficiency of the proposed method are demonstrated by a series of validation cases, including flow past stationary and moving cylinders, both prescribed and freely vibrating. The numerical method reproduces fairly well the vibration of an elastically mounted cylinder subjected to a fluid flow. In particular, vibration amplitude as well as vibration frequency are computed quite accurately. In addition, the predicted vortexinduced cylinder vibration lock-in regime is in good agreement with laboratory experiments.

\section{Introduction}

The topic of fluid-structure interaction (FSI) and specifically vortex-induced vibrations (VIV) is of critical importance in a number of engineering scenarios. Bridges, chimney stacks, marine cables and offshore riser pipes represent a selection of engineering structures that are known to undergo vibrations of significant amplitude when subjected to flows of air or water, often resulting in increased rates of fatigue damage with an associated risk of catastrophic failure. As a result, the topic has attracted increasing attention from the research community since the middle of the twentieth century.

Although many of the most important advances in our understanding of VIV have been achieved in the laboratory, the last 25 years have yielded numerous significant insights from numerical simulations. A number of excellent reviews of experimental and numerical progress exist in the literature, for example Sarpkaya (2004), Williamson and Govardhan (2004) and Williamson and Govardhan (2008), while the well-known books of Blevins (1990), Sumer and Fredsoe (1997) and Zdravkovich $(1997,2003)$ also constitute authoritative references. In this paper, the authors provide an appraisal and concise overview of previous work in FSI, first summarising the vast canon of experimental work that led to important early insights. They then place particular focus on simulationbased studies and the wide range of numerical techniques that have been applied therein since the early 1990s. The authors have chosen to focus on the classic case of flow past a single circular cylinder, as opposed to cylinders with other crosssectional shapes, as this is the most common practical application and has been by far the most studied arrangement to date. Finally, the authors present a numerical method to predict FSI and VIV and present results from a few selected case studies in which they simulate flow past a circular cylinder in various configurations.

\section{Vortex shedding and VIV}

\subsection{Flow past a stationary circular cylinder}

Flow past a stationary circular cylinder is known to become unstable when the cylinder Reynolds number exceeds 47 (Williamson, 1996). This instability gives rise to periodic shedding of vortices into the cylinder's wake, with a frequency of shedding $f_{\mathrm{s}}$. In the range $47<\operatorname{Re}<170-200$, where the upper limit depends on end conditions, the shedding is laminar and two dimensional (2D), and changes in Re are accompanied by significant changes in the Strouhal number, St $\left(=f_{\mathrm{s}} D / U\right)$. Williamson (1988a) observed that the onset of 3D shedding occurs by way of two distinct transitions. The first of these at $180 \leq \operatorname{Re} \leq 194$ involves the change from $2 \mathrm{D}$ shedding to mode A shedding and exhibits hysteresis, while the second, at $230<\operatorname{Re}<250$, entails a much more gradual shift from mode A 
to mode B shedding. Mode A is the name given by Williamson to denote $3 \mathrm{D}$ laminar shedding in which vortex loops, the wavelength of which scales on three to four diameters in the spanwise direction, appear in the wake as a result of the onset of deformation in the primary vortices in the near wake. The 3D structures associated with mode B shedding are much smaller, with a spanwise wavelength typically of about one diameter.

Increasing the Reynolds number still further, the authors encounter the various 'regimes' of vortex shedding that were first formalised by Roshko (1954) and then refined by Sumer and Fredsoe (1997). The distinct regimes are defined based on unique features of the cylinder wake and boundary layer: the lift and drag forces, $\boldsymbol{F}_{y}$ and $\boldsymbol{F}_{x}$, respectively, may also display markedly different behaviours under different regimes. A great deal of the concerted physical and numerical efforts of the late twentieth and early twenty-first centuries has focused on understanding vortex dynamics in the 'sub-critical' regime, which occupies the range $350<\operatorname{Re}<1 \times 10^{5}$ and is characterised by a fully turbulent wake and laminar boundary layers.

\subsection{Free and prescribed vibrations of rigid circular cylinders}

Three non-dimensional parameters are commonly used to characterise bluff-body free-vibration experiments: the normalised or 'reduced' velocity $\boldsymbol{U}_{\text {red }}\left(=\boldsymbol{U} / D f_{\mathrm{n}}\right)$, where $f_{\mathrm{n}}$ is the in vacuo natural frequency of the undamped oscillating system; the mass ratio, $m^{*}$, which is defined as the ratio of the mass of the cylinder, $m$, to the mass of the fluid displaced by it; and the structural damping ratio, $\beta$, which gives the ratio of the actual experimental damping coefficient, $c$, to the coefficient that would be required to achieve critical damping, $c_{0}$. In the seminal experiment of Feng (1968), an elastically mounted rigid circular cylinder was subjected to an air flow and permitted to move in the transverse direction only. The cylinder had a relatively high mass ratio of 248 , making it broadly representative of a chimney stack in air, for example, marine engineering situations such as flexible riser pipes are associated with lower mass ratios, of the order $O(1)$. By measuring the amplitude, $A$, and frequency, $f_{\mathrm{o}}$, of oscillation for a range of reduced velocities, Feng (1968) observed a phenomena known as 'lock-in', in which the vortex shedding synchronises with the natural frequency of the body, with the result that exaggerated amplitudes of oscillation are observed.

Feng (1968) observed peak amplitudes of oscillation of $\sim 0.53 \mathrm{D}$ and also a sharp drop in amplitude at a reduced velocity of $\sim 6 \cdot 4$. This discontinuous transition was observed by Feng (1968) to be hysteretic with increasing and decreasing reduced velocity, and has the effect of separating the amplitude response into 'branches' of response. Khalak and Williamson
(1999), in their experimental investigation of the transverse oscillations of a low mass ratio elastically supported circular cylinder in water, discovered three distinct divisions in the response: the 'initial', 'upper' and 'lower' branches.

The Skop-Griffin parameter, $S_{\mathrm{G}}\left(=2 \pi^{3} S_{t}^{2}\left(m^{*} \zeta\right)\right.$ ) (see Griffin, 1980), combines the mass ratio of an oscillating system with its damping ratio. Griffin collated a multitude of peak-amplitude data from various experiments and plotted them against $S_{\mathrm{G}}$ on $\log$ scales. The Griffin plot, as it has subsequently become known, is a useful format to present the effect of mass and damping ratio on peak response, although Govardhan and Williamson $(2000,2006)$ state that the degree of scatter that appears when the data are plotted on linear scales renders it somewhat less than definitive.

Govardhan and Williamson $(2000,2006)$ conducted transverse VIV experiments in water on a rig that allowed the damping ratio of the elastically supported rigid cylinder to be altered over a wide range of positive and negative values. The researchers discovered that the data do in fact collapse very well, with minimal scatter, if the Reynolds number is taken into account as an additional parameter in a 'modified Griffin plot'. Govardhan and Williamson therefore proposed that a re-normalised parameter, the 'modified amplitude', $A_{\mathrm{M}}\left(=A / A_{\zeta=0}\right)$, should henceforth be used, where $A$ is the actual transverse peak amplitude as measured in the experiment and $A_{\zeta=0}$ is the peak amplitude that would be expected for the case of zero damping at a given Reynolds number. By applying a functional fit to the data, the researchers arrived at an expression that permits the prediction of the peak amplitude of response of an elastically supported rigid circular cylinder, of given mass and damping ratios, as it undergoes transverse VIV.

Mass and damping effects are directly relevant to the type of branched VIV response envelope that Feng (1968) reported. Khalak and Williamson (1999) measured the amplitude response of an elastically damped rigid cylinder with a relatively low mass ratio $\left(m^{*}=10 \cdot 1\right)$ that was also constrained to oscillate only in the transverse direction, and drew useful comparisons to the response of Feng's (1968) cylinder. The low $m^{*}$ cylinder oscillated at much larger amplitudes, $A \approx 0 \cdot 95$, over a much wider range of reduced velocities, compared with Feng's (1968) cylinder $(A \approx 0 \cdot 6)$. By systematically investigating different combinations of $m^{*}$ and $\zeta$, thereby producing a range of mass-damping, Khalak and Williamson (1999) concluded that the peak amplitude of a transversely oscillating circular cylinder is in fact determined primarily by $m^{*} \zeta$, whereas the range of $\boldsymbol{U}_{\text {red }}$ for which non-negligible response occurs is governed principally by $m^{*}$ (when $m^{*} \zeta$ is constant). Two distinct types of amplitude response that are dependent entirely on the mass-damping of the cylinder were also identified. Essentially, 
it was found that the amplitude response of low $m^{*} \zeta$ cylinders, in addition to achieving larger peak amplitudes and occurring over a wider range of reduced velocities, comprises the initial, upper and lower branches, whereas high $m^{*} \zeta$ responses incorporate only the initial and lower branches. It was also shown that the phase jump of $180^{\circ}$ that had previously been observed by Ongoren and Rockwell (1988) when the forcing frequency in their controlled oscillation experiment surpassed the vortex-shedding frequency, actually accompanies the transition between the upper and lower branches for a low $m^{*} \zeta$ transversely oscillating cylinder. Ongoren and Rockwell (1988) reported that this phase jump results from a change in the timing of vortex shedding.

Another important observation made by Khalak and Williamson (1999) concerned the departure of the ratio $f_{\mathrm{o}} / f_{\mathrm{n}}$ in the lock-in region from unity. Although the experiments of Feng (1968) had indicated that in this region $f_{\mathrm{o}} / f_{\mathrm{n}}$ remains approximately constant, Khalak and Williamson (1999) showed that this behaviour is typical only for high $m^{*} \zeta$ cylinders and that $f_{\mathrm{o}} / f_{\mathrm{n}}$ can, in fact, be significantly larger than unity when $m^{*} \zeta$ is low (values up to 1.4 were recorded). Williamson and Govardhan (2004) therefore proposed to define lock-in as 'the matching of the frequency of the periodic wake vortex mode with the body [natural] oscillation frequency [in stationary fluid]'.

Building on Lighthill's hypothesis that it is possible to decompose the total fluid force, $\boldsymbol{F}_{\text {total }}$, into potential force, $\boldsymbol{F}_{\text {potential }}$, and vortex force, $\boldsymbol{F}_{\text {vortex }}$, components (Lighthill, 1986), Govardhan and Williamson (2000) introduced a vortex phase, $\phi_{\text {vortex }}$, defined as the phase by which the vortex force leads to the cylinder displacement, and proposed a modified version of the equation of motion in which only the vortex force is considered. By applying this definition, the researchers showed that the transition between the initial and upper branches of response involves a jump in $\phi_{\text {vortex }}$ and a change in shedding mode. The transition from the upper to lower branch, however, is characterised by a jump in $\phi$, the phase by which the total force leads to the displacement, but no change in shedding mode.

Three essentially equivalent variations exist for prescribed oscillation experiments: a cylinder forced to oscillate sinusoidally transverse to a steady incident fluid flow; a cylinder towed on a sinusoidal trajectory through an undisturbed stationary fluid and a stationary cylinder subjected to an oscillatory flow. The principal motivation for performing tests of this nature arises from the inherent reduction in parameter space compared with free-vibration experiments. Essentially, the experimenter is free to vary the amplitude and frequency of the oscillations over a range governed by the limitations of the equipment and/or his/her area of interest.
Williamson and Roshko (1988) performed a very detailed study of the wake patterns of a circular cylinder that was being towed on a sinusoidal trajectory through an undisturbed stationary fluid. The Reynolds number of the flow past the cylinder varied in the range $300 \leq \mathrm{Re} \leq 1000$. The amplitude, $A / D$, and the wavelength, $\gamma / D$, of the motion were systematically varied such that a $(\gamma, A)$ domain defined by $(1 \leq \gamma / D \leq 15$, $0 \cdot 1 \leq A / D \leq 5 \cdot 0)$ was investigated. Five distinct modes of vortex shedding were recorded; the $2 \mathrm{~S}, 2 \mathrm{P}$ and $\mathrm{P}+\mathrm{S}$ modes, a coalesced $2 \mathrm{~S}$ mode, $\mathrm{C}(2 \mathrm{~S})$ and a coalesced $\mathrm{P}+\mathrm{S}$ mode, $\mathrm{C}(\mathrm{P}+\mathrm{S})$, as well as a region in which no synchronised shedding pattern was discernible. In the $2 \mathrm{~S}$ mode, two single vortices are shed from the cylinder surface in each oscillation cycle, while two pairs of vortices are shed per cycle in the $2 \mathrm{P}$ mode. $\mathrm{P}+\mathrm{S}$ shedding is associated with an asymmetrical wake, as the cylinder sheds one single vortex and one vortex pair per oscillation cycle. The $\mathrm{C}(2 \mathrm{~S})$ and $\mathrm{C}(\mathrm{P}+\mathrm{S})$ labels refer to vortex wakes in which the respective $2 \mathrm{~S}$ and $\mathrm{P}+\mathrm{S}$ shedding modes occur initially, only for individual vortices to coalesce with each other in the near wake to form larger vortex structures which then propagate downstream. Govardhan and Williamson (2000) overlaid the response of their free-to-oscillate low $m^{*} \zeta$ cylinder on a portion of this map, and the agreement with the modes that they observed using digital particle image velocimetry (DPIV) was very good, particularly regarding the transition between initial to upper branches.

Gopalkrishnan (1993) and Staubli (1983) measured lift and drag forces acting on a cylinder undergoing sinusoidal oscillations in flows of $\mathrm{Re}=10^{4}$ and $6 \times 10^{4}$, respectively, separating the total lift $\left(C_{\mathrm{L}}\right)$ and drag $\left(C_{\mathrm{D}}\right)$ forces into components varying at the cylinder oscillation frequency, $C_{\mathrm{Lo}}$ and $C_{\mathrm{Do}}$, and those oscillating at the vortex-shedding frequency, $C_{\mathrm{Ls}}$ and $C_{\text {Ds. }}$ Particular attention was given by both experimenters to the lift coefficients in-phase with the cylinder velocity and acceleration, $\quad C_{\mathrm{Lo}_{v}}\left(=C_{\mathrm{Lo}} \sin \phi_{\mathrm{o}}\right)$ and $C_{\mathrm{Lo}_{a}}\left(=C_{\mathrm{Lo}} \cos \phi_{\mathrm{o}}\right)$, respectively. These quantities are of interest due to the information that they can bring about energy transfer between the fluid and the cylinder. Essentially, a positive value of $C_{\mathrm{Lo}_{v}}$ represents energy transfer to the body by the fluid while $C_{\mathrm{Lo}_{v}}$ is negative energy transferred from the body to the fluid. More recently, Morse and Williamson (2009) carried out a very thorough parametric study in which a cylinder underwent prescribed sinusoidal oscillations transverse to a flow of $\mathrm{Re}=$ 4000. The frequency-amplitude domain that they explored was defined by the ranges $0 \leq A_{y} / D \leq 1.6$ and $0.06 \leq f_{\mathrm{o}} D / U \leq 0.5$ and comprised an extremely fine stencil of data points. By considering a very accurately drawn contour of zero fluid excitation (essentially their $C_{\mathrm{Lo}_{v}}=0$ contour), they showed that the hysteresis between the initial and upper branches observed in previous free-vibration experiments (Khalak and Williamson, 1999) is, in fact, caused by the concave shape of this contour. 


\section{Numerical simulation of flows past circular cylinders and VIV}

Low Reynolds number 2D flow past a single circular cylinder has been simulated numerically by many researchers since the appearance of a handful of pioneering studies in the 1970s. As with other simulations of canonical flows, many numerical studies of 2D cylinder flow have been motivated by the desire to validate a new method or code against a case that involves challenging features such as separation and unsteadiness, and for which reliable experimental data are readily available for comparison. Other studies, however, have sought to increase one's understanding of vortex dynamics and transition to three dimensionality and turbulence, which is an important engineering flow case in its own right.

The immersed boundary method (IBM), which was introduced by Peskin and co-workers (Peskin, 1972, 1977) and is being employed later in this paper, has been successfully applied to cylinder flows by a number of researchers who aimed to demonstrate the ability of their implementations of, and variations on, the IBM to predict accurately and reliably flows past bluff bodies. The basic premise of the IBM is to compute the fluid flow around solid boundaries on a Cartesian grid and account for the solid that is embedded (or immersed) inside the grid through an appropriate methodology that ensures that the no-slip condition on the boundary is maintained. The IBM on Cartesian grids offers particular advantages over bodyconforming grids for flow domains with singularities and/or moving boundaries for which preservation of high-quality body-conforming grids is difficult or impossible to achieve. In this way, the method maintains the computational efficiency and simplicity of Cartesian grids while permitting the simulation of complex and/or moving geometries. Furthermore, the IBM allows computation of a body's movement in the fixed Eulerian frame of reference, negating the need to introduce a secondary frame that moves with the body (the 'body-fixed' frame), thereby circumventing the need to perform conversions between more than one frame of references. Several different variants of the IMB method have evolved since the 1970s, which can be grossly divided into direct or feedback forcing methods (Fadlun et al., 2000; Kempe and Fröhlich, 2012; Kim et al., 2001; Lai and Peskin, 2000; Shin et al., 2008; Tseng and Ferziger, 2003; Uhlmann, 2005a; Yang and Balaras, 2006; Yang et al., 2009; Yu and Shao, 2007), sharp-interface cut-cell methods (Seo and Mittal, 2011; Udaykumar et al., 2001) and hybrid versions of the two (Gilmanov and Sotiropoulos, 2005). Regarding circular cylinder flows, the studies of Borazjani and Sotiropoulos (2009), Gazzola et al. (2011), Kim and Choi (2006), Kolomenskiy and Scheider (2009) and Yang et al. (2008) constitute a selection of studies in which flow past various arrangements of single or tandem cylinders that were either stationary, freely vibrating or a combination of the two, have been investigated using the IBM. The IBM continues to grow in popularity as a result of the considerable advantages it offers over body-fitted approaches, and can be considered as a stateof-the-art numerical method for the computation of complex FSI problems. For comprehensive reviews of developments in the IBM, the interested reader may refer to Iaccarino and Verzicco (2003), Peskin (2002) and Sotiropoulos and Yang (2014).

\subsection{Stationary cylinder flows}

Norberg (2003) presented a comprehensive collection of some of the key quantities that were observed in numerical simulations of flows past stationary circular cylinders in the period 1972-2003. Two-dimensional simulations of low Reynolds number flows $(\operatorname{Re}<500)$ and $3 \mathrm{D}$ simulations of higher Reynolds number flows $\left(\operatorname{Re}<10^{5}\right)$ were collated in two tables that gave details of the Reynolds number of the flow, the computational domain, the solution method and the oscillating lift coefficient that was observed. For the $3 \mathrm{D}$ experiments that used large eddy simulations (LES), the level of subgrid-scale turbulence dissipation used was also reported in the guise of a Smagorinsky constant. The list of low Re simulations has subsequently been extended by some notable recent applications of the IBM: Kempe and Fröhlich (2012), Kim et al. (2001), Lai and Peskin (2000), Shin et al. (2008), Tseng and Ferziger (2003), Uhlmann (2005a), Yang and Stern (2012), Yang et al. (2009), and $\mathrm{Yu}$ and Shao (2007) all employed variations of the direct forcing method to simulate interaction between moving bodies in the fluid, choosing to validate the accuracy of the method on flow past a stationary circular cylinder. Fadlun et al. (2000), on the other hand, chose to validate their approach using the case of flow past a stationary sphere and in doing so demonstrated formal second-order accuracy and a more accurate distribution of body forces to neighbouring grid points in the fluid domain than had previously been achieved with the IBM.

Most 3D computations that have been performed to date have employed periodic boundary conditions at the pipe ends, so that the length of the pipe, $L_{z}$, also represents the length over which the simulated flow is periodic. Henderson (1997) noted that the normalised pipe length in $3 \mathrm{D}$ simulations, $L_{z} / D$, does not directly correspond to the length-to-diameter ratio of pipes in physical experiments, and the two quantities should not be regarded as equivalent. The parameter $L_{z} / D$ in $3 \mathrm{D}$ simulations has been shown to exert a considerable influence on the behaviour of the lift force. Norberg (2003) concluded that at the time of writing it was not possible to ascertain the precise value of $L_{z} / D$ that would be required for any given flow past a circular cylinder such that all of the necessary flow features would be computed.

Early LES simulations of circular cylinder flows were performed at Stanford University by Beaudan and Moin (1994), Kravchenko and Moin (1998), and Mittal and Moin (1997), focusing now on the classic $\mathrm{Re}=3900$ benchmark case. 
Although the simulations showed good agreement with experimental data in terms of mean velocity profiles and Reynolds stress distributions in the far wake, agreement in the near wake was less satisfactory. Furthermore, Beaudan and Moin (1994), in particular, suffered from excessive numerical dissipation, and therefore overdamped power spectra, due to their upwind scheme. Mittal and Moin's (1997) central difference scheme improved on this, producing better agreement with experiments with respect to power spectra in the wake. Breuer (1998) focused exclusively on key numerical issues known to affect the quality of LES computations, also using $\mathrm{Re}=3900$ flow past a circular cylinder as the benchmark. He found that central difference schemes of second- or fourthorder accuracy in space are well suited, compared with more diffusive upwinding approaches. The influence of the subgridscale model was also investigated, and the now well-known dynamic model of Germano et al. (1991) was found to give the best performance. The importance of adequate spanwise resolution for computations of 3D shedding was also highlighted.

Dong and Karniadakis (2005) conducted direct numerical simulations (DNS) of flow past a stationary cylinder and a cylinder undergoing prescribed oscillations at $\mathrm{Re}=10000$, using a spectral/hp method to discretise the flow field in the streamwise-crossflow planes and a Fourier spectral expansion in the spanwise direction, an approach that was first introduced by Henderson and Karniadakis (1995). To date, $\mathrm{Re}=10000$ remains the highest Reynolds number at which flow past a circular cylinder has been simulated using the DNS. The numerical results have shown good agreement with experiments in terms of the Strouhal number and the mean drag, base pressure and lift coefficients, while vortex dynamics in the near wake were in good agreement with PIV visualisations of the corresponding case. Dong et al. (2006) performed simulations of flow past a circular cylinder at $\mathrm{Re}=3900$ and 10000 and, together with complementary PIV measurements, used the computed flow fields to investigate shear layer statistics in this transitional regime. The results showed that elevated Reynolds stresses occur further upstream at higher Reynolds numbers, hinting at earlier onset of shear layer transition. In general, it was found that patterns of all of the key quantities that characterise the near wake and separating shear layers (i.e. spanwise vorticity, fluctuating velocity field) move closer to the base of the cylinder as Re is increased.

Breuer (2000) pushed the LES approach to much higher Reynolds numbers than had previously been achieved in his computations of flow past a cylinder at $\mathrm{Re}=140000$. Encouraging agreement was observed between the simulations and the experimental data of Cantwell and Coles (1983), particularly in the near wake, although curiously the agreement was not improved by increased mesh resolution.
In the last 20 years, a number of numerical studies have sought to build on the experimental advances that Williamson (1988a) made in understanding the onset of three dimensionality in vortex shedding from circular cylinders. Barkley and Henderson (1996) published a seminal study in which they performed linear stability analysis of the periodic wake behind a cylinder for Reynolds numbers in the range $140 \leq \operatorname{Re} \leq 300$. Using a derivative of the spectral/hp element method introduced by Henderson and Karniadakis (1995), they obtained DNSs of the time-varying flow field in the cylinder wake on 2D domains, before determining the stability of these fields to $3 \mathrm{D}$ perturbations using Floquet stability analysis. The $2 \mathrm{D}$ wake was first observed to become linearly unstable at $\mathrm{Re}=188.5 \pm 1 \cdot 0$, and a second instability occurred at $\mathrm{Re}=259$. The respective spanwise length scales associated with these bifurcations were 3.96 and 0.822 cylinder diameters; the Floquet analysis therefore agreed very convincingly with the mode A and mode B instabilities that were observed experimentally by Williamson (1988a). Sheard et al. (2005) also conducted detailed Floquet analysis to address the conjecture that had arisen regarding the existence of a possible subharmonic instability, now known as mode $\mathrm{C}$, between the two previously defined modes.

\subsection{Free and prescribed vibrations of rigid circular cylinders}

Numerical simulations of the VIV of elastically supported rigid circular cylinders have for the most part been focused on low Reynolds number flows, principally due to limitations imposed by the computing resources. A number of studies have attempted to replicate the experiments of Anagnostopoulos and Bearman (1992), in which an elastically mounted cylinder of very high mass ratio, $m^{*} \approx 148$, and low damping, $1.20 \times$ $10^{-3} \leq \zeta \leq 1 \cdot 52 \times 10^{-3}$ was permitted to oscillate transversely to an oncoming laminar flow. The flow speed was altered such that the Reynolds number range $90 \lesssim \operatorname{Re} \lesssim 150$ was explored. The peak amplitude, $A / D \approx 0 \cdot 55$, was significantly lower than has been observed in similar, higher Reynolds number experiments (see e.g. Govardhan and Williamson, 2000; Khalak and Williamson, 1999), prompting Govardhan and Williamson (2000) to speculate that this was a Reynolds number effect, brought about by a suspected inability of cylinders oscillating in laminar flows to achieve the upper branch response.

Although numerical simulations of the case investigated experimentally by Anagnostopoulos and Bearman (1992) have generally yielded peak response amplitudes that are comparable with the experimentally observed value, they have struggled to match the range of reduced velocities across which large amplitude responses were recorded. Nomura (1993), Schulz and Kallinderis (1998), and Wei et al. (1995), all employed variations on the arbitrary Lagrangian-Eulerian technique to cope with moving body-fitted 2D grids, while Li et al. (2002) used a spectral element fluid solver in a translating frame of reference, 
thereby negating the need for dynamic mesh regeneration. In all cases, the peak amplitude of vibration was in reasonable agreement with the experiments but the reduced velocity at which the peak occurred and the range of reduced velocities over which fluid dynamic excitation was observed were somewhat less so. Yang and Balaras (2006) and Yang et al. (2008) also chose these experiments as a validation benchmark for their IBM approach, which employed momentum forcing to reconstruct the velocity field near the cylinder surface without smearing the sharp interface and introduced the concept of field extension to deal with the potentially problematic issue of freshly cleared grid cells or points (i.e. those that change from a solid cell or point to a fluid cell or point in two successive time steps). As with previous numerical studies, the peak amplitude of response was close to the experimental value but the extent of the response envelope in terms of reduced velocity was not as well matched.

Yang et al. (2008) also presented comparisons against the computations of Leontini et al. (2006), who used a spectral element solver with a moving frame of reference in a way similar to Li et al. (2002) to investigate VIV of a 2D cylinder with relatively low mass and damping ratios $\left(m^{*}=10, \beta=0 \cdot 01\right)$ at $\mathrm{Re}=200$. An important outcome of the study was the occurrence of the type of branching behaviour that had been previously observed only at higher Reynolds numbers (Feng, 1968; Khalak and Williamson, 1999; Willden, 2006). Yang et al. (2008) were able to match the responses of Leontini et al. (2006) very accurately.

The problem of freshly cleared grid cells or points is in fact common to all IBMs where moving bodies are involved. The occurrence of freshly cleared cells provokes a violation of mass conservation properties (Seo and Mittal, 2011), and thus pressure and velocity discontinuities across the immersed boundary, which leads to spurious pressure and velocity oscillations and to inaccuracy or instability of the numerical scheme. Several remedies to this problem have been proposed, depending on the method (e.g. Seo and Mittal (2011) for sharp interfaces, and Peskin (1977) and Uhlmann (2005a) for distributed forcing methods). Yang et al. (2009) used smoothed discrete delta functions to reduce non-physical oscillations that occur in freshly cleared cells, replicating the case of a cylinder undergoing prescribed transverse oscillations in $\mathrm{Re}=185$ flow that had previously been investigated by Uhlmann (2005a) and Guilmineau and Queutey (2002), as well as the free VIV case of Leontini et al. (2006); the resulting time traces of lift and drag forces were characterised by noticeably fewer oscillations than when non-smoothed functions were used.

Shiels et al. (2001) conducted simulations of a cylinder that was free to vibrate in the transverse direction in a flow of
$\operatorname{Re}=100$ using a viscous-vortex method, with a cylinder of zero mass, stiffness and damping. The cylinder was therefore free to move without restraint in the transverse direction, and the vortex force was always balanced by the added mass force: it was noted that this balance was achieved through a constant mutual adjustment between the body motion and the trajectories of vorticity in the boundary layers. Remarkably, the cylinder proceeded to oscillate with a large amplitude $(A / D=0 \cdot 47)$ at a frequency close to the $\mathrm{Re}=100$ stationary cylinder shedding frequency, $f_{\mathrm{o}} D / U=0 \cdot 156$.

Hysteretic responses of the type that have been observed in laboratory experiments (Feng, 1968; Khalak and Williamson, 1999) have relatively recently been encountered in numerical simulations. Singh and Mittal (2005), for instance, simulated a $\operatorname{Re}=100$ flow past a circular cylinder $\left(m^{*}=10\right)$ that was free to move in both the in-line and transverse directions, and observed hysteretic responses in both directions. Singh and Mittal (2005) also investigated the effects of altering the Reynolds number of their flow, in the range $50 \leq \operatorname{Re} \leq 500$, using the correlating effect of lock-in (Toebes, 1969) to justify their $2 \mathrm{D}$ simulations in the $3 \mathrm{D}$ regime. For $\mathrm{Re}>300, \mathrm{P}+\mathrm{S}$ shedding was observed for the first time in free oscillation simulations or experiments.

Willden (2006) solved the velocity-vorticity formulation of the 2D Navier-Stokes equations: the fluid solver was loosely coupled to a mass-spring-damper model of a circular cylinder that was free to vibrate in the transverse direction only. The flow was computed in the body-fixed frame of reference rather than the absolute frame for reasons of computational efficiency and the flow Reynolds number was $10^{4}$; a Smagorinskytype model was used to account for the effects of subgrid-scale turbulent stresses. The correlating effect of lock-in was used to justify the use of $2 \mathrm{D}$ simulations. The key outcomes of the investigation involved the appearance of $2 \mathrm{P}$ and $\mathrm{P}+\mathrm{S}$ shedding modes on the lower branch, and $2 \mathrm{~S}$ on the initial branch. Discrepancies with experimental observations regarding the nature of the $2 \mathrm{P}$ shedding on the upper branch, and the variation of shedding frequency on that branch, led the author to conclude that 2D approaches are incapable of correctly computing the upper branch response, which is known to entail significant three dimensionality (Hover et al., 2004). This conclusion was in agreement with the complementary DNS and experimental study of Blackburn et al. (2001).

Willden and Graham (2006) conducted simulations of flow past cylinders whose mass ratios varied in the range $1 \leq m^{*} \leq 50$, for Reynolds numbers that extended to the $3 \mathrm{D}$ shedding regime, once again using the correlating effect of lock-in to justify $2 \mathrm{D}$ computations. Using the limiting case of zero structural damping, it was shown that three distinct regimes of response are possible; the higher mass ratio cylinders responded in the 
classical manner close to resonance, whereas the intermediate mass ratio cylinders displayed a tendency for super-harmonic excitation and the lowest mass ratio cylinders $\left(m^{*}=1\right)$ responded infinitely, that is to say its response envelope did not appear to have an upper limit of reduced velocity. Other researchers to apply 2D simulations for cylinders ostensibly oscillating in the 3D shedding regime include Charles Dalton's group at the University of Houston: Al-Jamal and Dalton (2004), Lu and Dalton (1996), and Zhang and Dalton (1996), simulated circular cylinder VIV at Reynolds numbers up to 13000 , with reasonable agreement to experiments.

As has been the case in physical experiments, a number of numerical studies have attacked the problem of VIV by simulating cylinders undergoing oscillations of prescribed amplitudes and oscillations. As stated previously, this introduces a favourable reduction in the parameter space while still producing wake synchronisation that is equivalent to that which occurs in free vibrations. In the numerical simulations of Meneghini and Bearman (1995), the high-amplitude oscillatory flow about a circular cylinder was assumed to be equivalent to cylinder oscillations in a steady flow. They used a discrete vortex method with viscous diffusion to simulate $\mathrm{Re}=200$ flow past a cylinder, and varied the amplitude and frequency of oscillation in the ranges at which lock-in had previously been observed in experiments. Particular attention was paid to the locking-in of the vortex-shedding frequency to the oscillation frequency; at low amplitudes of oscillation the lock-in range extended over a much larger range of frequencies below the Strouhal frequency than above it. An asymmetric wake, produced by $\mathrm{P}+\mathrm{S}$ shedding, was observed in the wake for amplitudes larger than about 0.6 cylinder diameters. Udaykumar et al. (2001) replicated the computations of Meneghini and Bearman (1995) to demonstrate the ability of their sharp-interface IBM approach that attacked the aforementioned problem of freshly cleared cells by temporarily merging freshly cleared cells with neighbouring cells. The approach, which also made use of a multigrid method for the solution of the pressure Poisson equation, showed good agreement with the computations of Meneghini and Bearman (1995). Both studies confirmed the occurrence of $\mathrm{P}+\mathrm{S}$ type shedding at high amplitudes of oscillation.

The 2D DNS of Dong and Karniadakis (2005), performed with their spectral/hp code, showed very impressive levels of agreement with the experiments of Gopalkrishnan (1993). Due to the substantial computational expense of the simulations the most highly resolved case required 250000 central processing unit hours - only four oscillation frequencies and one amplitude $\left(f_{\mathrm{o}} D / U=14,0 \cdot 17,0 \cdot 21,0 \cdot 25\right.$ and $\left.A_{y} / D=0 \cdot 3\right)$ were computed. Comparisons between the numerical and experimental results, in terms of the oscillating lift coefficient, the mean drag coefficient, the phase angle, the lift coefficient inphase with the cylinder's velocity, were very encouraging.
Given that much of the research effort in circular cylinder VIV is motivated by the need for better understanding of the destructive oscillatory responses of long structures such as marine cables and riser pipes, many researchers have placed particular emphasis on spanwise features. In the case of tensioned marine riser pipes, the structures have extremely high length-to-diameter ratios and are known to experience multimodal responses, with very high spanwise mode numbers being excited. This is a particularly difficult challenge, both experimentally and numerically, and as a result very few data sets exist for VIV of flexible structures that have aspect ratios comparable with those used in the field. Chaplin (2005) presented results from a very ambitious exercise in which blind simulations from 11 different numerical models were compared with laboratory tests. In the tests, a long, cylindrical pipe with a length-to-diameter ratio of $\sim 469$ was subjected to a stepped current and VIV was observed. Four of the numerical models used CFD to predict the forces on discrete $2 \mathrm{D}$ simulation planes positioned along the pipe's length: this approach is known as strip theory and is based on the assumption that in lock-in the flow is locally 2D (Toebes, 1969). The other models were based on empirical knowledge of VIV responses. In the CFD-based models, the computed hydrodynamic forces acting on the 2D cylinder sections are then applied to structural dynamics models of the pipe, which are then allowed to respond before new displacements at a fractional time step are used to move the $2 \mathrm{D}$ cylinder sections to the corresponding new positions in the CFD strips, completing the two-way FSI. The method is strictly valid only during lock-in, but since fully 3D simulations of flows past such long bodies are well beyond current computing capabilities, strip theory is considered to be arguably the most pragmatic option at present. In general, the CFD-based models under-predicted the cross-flow response of the pipe while over-predicting the in-line displacement. The curvatures predicted by CFD codes varied between 25 and $115 \%$ of the measured values in the cross-flow directions, a result that has considerable significance because fatigue damage in such pipes is known to be directly proportional to the curvature.

Among other numerical researchers to study spanwise effects in flows past 3D cylinders, Newman and Karniadakis (1996, 1997) presented results from DNS of $\mathrm{Re}=100,200$ and 300 flows past flexible cables that were assumed to be infinitely long and periodic. Prescribed and free vibrations were compared; transition to $3 \mathrm{D}$ vortex shedding was observed at a lower Reynolds number in VIV than in prescribed oscillations. Increased in-line and transverse separation between vortex pairs were observed in the wakes that occur at the spanwise anti-nodes of vibration compared with those at the nodes. Newman and Karniadakis (1997) paid particular attention to spanwise trends, and observed both standing and travelling wave responses. The term 'standing wave' refers to an 
oscillation in which the spanwise position of the nodes does not change as a function of time. Travelling waves, by contrast, are characterised by the steady progression of nodes and antinodes along the pipe and may involve oblique vortex shedding, whereby the axes of the shed vortices are not parallel to that of the cable (Williamson, 1989).

\section{An IBM-based numerical method to predict FSI and VIV}

In the following, we present a numerical method, specifically developed for a (reasonably) accurate and stable calculation of VIV. The method is based on a standard Navier-Stokes solver, employs an IBM for the representation of the structure, including various refinements to the original IB method, and features a strongly coupled FSI algorithm (illustrated in Figure 1). In the case of a moving body, the fluid flow and body motion are computed in the same fixed frame of reference, without the need for separate absolute and body-fixed frames.

\subsection{Fluid solver}

The governing equations that describe the motion of an incompressible fluid are (e.g. Rodi et al., 2013)

1. $\frac{\partial \boldsymbol{u}}{\partial t}+\nabla \boldsymbol{u} \boldsymbol{u}=-\nabla p+\frac{1}{\mathrm{Re}} \nabla^{2} \boldsymbol{u}+\boldsymbol{f}$

2. $\nabla \boldsymbol{u}=0$

where $\boldsymbol{u}$ is the velocity vector, $t$ is the time, $p$ is the pressure and $\boldsymbol{f}$ an external forcing term used to enforce the no-slip condition on immersed boundaries in a Cartesian grid. Equations 1 and 2 are non-dimensionalised using a free stream velocity $\boldsymbol{U}_{\infty}$ and a length scale; here the cylinder diameter is $D$. The Reynolds number is defined as $\operatorname{Re}=D \boldsymbol{U}_{o} d v$, in which $v$ is the kinematic viscosity of the fluid. The fluid solver is based on the LES code Hydro3D, a finite-difference-based Cartesian grid solver that has undergone

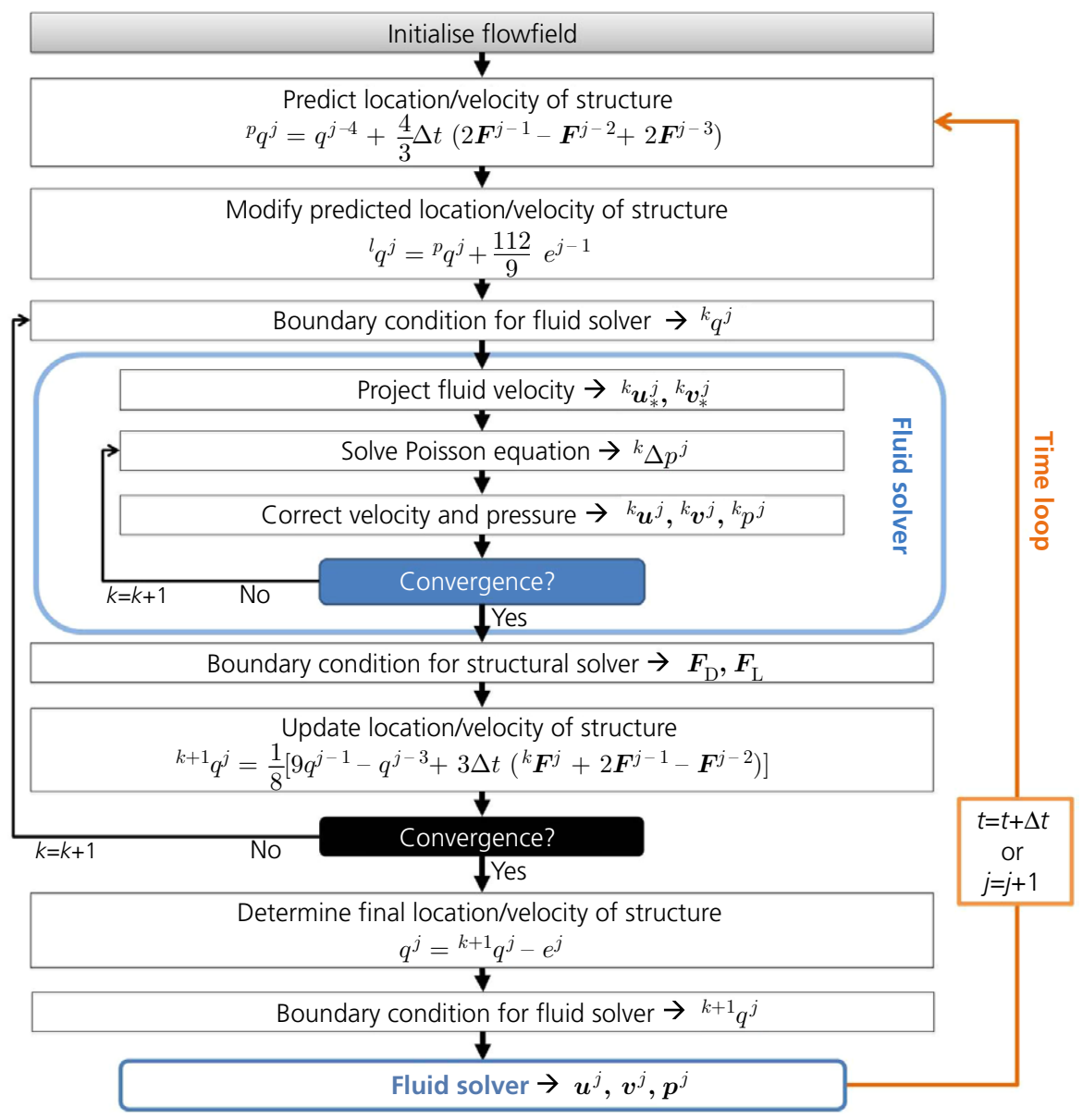

Figure 1. Flowchart of the numerical procedure strongly coupling the fluid and structural solvers 
excessive validation for different flows (e.g. Bomminayuni and Stoesser, 2009; Kara et al., 2012a; Kim and Stoesser, 2011; Kim et al., 2013; Papanicolaou et al., 2012). The code uses the diffuse direct forcing approach of Uhlmann (2005a), in which forces between Lagrangian marker points, representing the immersed body, and Eulerian (fluid) grid points are transferred by a discrete delta function. The discrete form of the momentum equation that includes a direct forcing term to satisfy the boundary conditions of the immersed boundary is written as

3. $\frac{\boldsymbol{u}^{n+1}-\boldsymbol{u}^{n}}{\Delta t}=(\mathrm{rhs})^{n+1 / 2}+\boldsymbol{f}^{n+1 / 2}$

where rhs contains convective, viscous and pressure gradient terms at some intermediate time step $n+1 / 2$ and $\Delta t$ is the discrete time step. The direct forcing term $f$ is calculated explicitly (directly) and imposes the boundary conditions at locations in the domain where the structure and the fluid have the same velocity, $v$, as

4. $\boldsymbol{f}^{n+1 / 2}=\frac{\boldsymbol{v}^{n+1}-\boldsymbol{u}^{n}}{\Delta t}-(\mathrm{rhs})^{n+1 / 2}$

Since these locations seldom coincide with the Eulerian grid points of the fluid, the Eulerian force needs to be transferred to and from Lagrangian locations; that is, locations on the (moving) immersed boundary. This is accomplished by the regularised delta functions, as initially proposed by Peskin $(1972,1977)$ which is used as follows

5. $f_{i}^{n+1 / 2}=\sum_{L=1}^{N_{L}} \boldsymbol{F}^{n+1 / 2}\left(X_{L}\right) \delta_{h}\left(x_{i}-X_{L}\right) \Delta V_{L}$

in which the capital letters refer to quantities that are computed at Lagrangian locations, $X_{L}$, and the small letters refer to quantities at Eulerian locations, $x_{i} . \boldsymbol{F}$ is the force at the selected number of Lagrangian locations in space, $N_{L}$, and $V_{L}$ is an assigned volume around each location, which is of the order of the cube of the Eulerian grid spacing, $h$. Here, the smooth regularised delta function due to Yang et al. (2009) is used and the Lagrangian force is computed according to Uhlmann (2005a)

6. $\boldsymbol{F}^{n+1 / 2}\left(X_{L}\right)=\frac{\boldsymbol{V}-\tilde{\boldsymbol{U}}\left(X_{L}\right)}{\Delta t}$

with

7. $\tilde{\boldsymbol{U}}\left(X_{L}\right)=\sum_{x \in g_{h}} \tilde{\boldsymbol{u}}(x) \delta_{h}\left(x-X_{L}\right) h^{3}$

where $\boldsymbol{V}$ is the desired velocity; that is, the immersed boundary's velocity and $\tilde{\boldsymbol{u}}, \tilde{\boldsymbol{U}}$ are intermediate velocities at Eulerian and Lagrangian forcing points, respectively. Equation 5 is evaluated at a collection of Cartesian grid points, $g_{h}$, in the vicinity of the immersed boundary.

In Hydro3D, the convection and diffusion terms are approximated using second-order central differences and time integration is accomplished by a semi-implicit Runge-Kutta/ Crank-Nicholson scheme, which is used to project the velocity. A Poisson equation for the pressure correction $\Delta p$ is solved iteratively by an efficient multi-grid technique (Bunner, 1998) and $\Delta p$ is then used to obtain the pressure and to correct the projected velocity.

\subsection{Structure solver}

In this study, flow-induced vibrations, specifically transverse oscillations of a circular cylinder due to vortex shedding, are to be predicted numerically. This can be approximated as a massspring-damper system of mass $m$, with damper coefficient $c$ and spring constant $k$. The non-dimensional form of the equation of motion, which describes the movement of the structure that is displaced transversely to the flow as a result of fluid forces and structural properties, is an ordinary differential equation and is written as (e.g. Yang et al., 2008)

8. $\ddot{y}^{*}+2 \xi\left(\frac{2 \pi}{\boldsymbol{U}_{\text {red }}}\right) \dot{y}^{*}+\left(\frac{2 \pi}{\boldsymbol{U}_{\text {red }}}\right)^{2} y^{*}=\boldsymbol{a}_{y}^{*}$

where $\dot{y}$ and $\ddot{y}$ denote the first and second derivatives with respect to a dimensionless time $t^{*}$ of the dimensionless displacement $y^{*}=y(t) / D$ in the $y$-direction of a cylinder of diameter D. $\boldsymbol{U}_{\mathrm{red}}=\boldsymbol{U}_{\infty} /\left(f_{\mathrm{N}} D\right)$ is the reduced velocity in which $f_{\mathrm{N}}=(1 / 2 \pi) \sqrt{k / m}$ is the natural frequency of the cylinder. $\xi=c /(2 \sqrt{\mathrm{km}})$ expresses the damping and $\boldsymbol{a}_{y}^{*}$ is the acceleration due to lift forces, calculated as

9. $\quad \boldsymbol{a}_{y}^{*}=\frac{2 c_{\mathrm{L}}}{m^{*} \pi}$

where $c_{\mathrm{L}}$ is the coefficient of lift and $m^{*}=m / m_{\mathrm{f}}$ is the dimensionless mass with $m_{\mathrm{f}}$ being the fluid mass that is being displaced by the cylinder.

\subsection{Strong coupling}

At every instant in time the fluid solver requires boundary conditions regarding the position and velocity of the structure; similarly, the structure solver requires the acting instantaneous fluid forces and hence the two systems require strong coupling. This is achieved by using Hamming's fourth-order predictorcorrector method (Carnahan et al., 1969) and Equation 8 can be rewritten as

10. $\dot{\mathbf{q}}(t)=\boldsymbol{F}(t)$ 
where

11. $\dot{\mathbf{q}}(t)=\left[\begin{array}{c}\frac{\mathrm{d} y^{*}(t)}{\mathrm{d} t} \\ \frac{\mathrm{d} v^{*}(t)}{\mathrm{d} t}\end{array}\right]$

and

12. $\quad \boldsymbol{F}(t)=\left[\begin{array}{c}v^{*}(t) \\ a_{y}(t)-(2 \xi \omega) v^{*}(t)-\omega^{2} y^{2}(t)\end{array}\right]$

which expresses the history of the motion of the structure and its load due to external forces. The integration of Equation 12 in time is achieved by the procedure proposed by Preidikman and Mook (2000), using a predictor

13. ${ }^{p} q^{j}=q^{j-4}+\frac{4}{3} \Delta t\left(2 \boldsymbol{F}^{j-1}-\boldsymbol{F}^{j-2}+2 \boldsymbol{F}^{j-3}\right)$

a modifier

14. ${ }^{l} q^{j}={ }^{p} q^{j}+\frac{112}{9} e^{j-1}$

and a corrector

15. ${ }^{k+1} q^{j}=\frac{1}{8}\left[9 q^{j-1}-q^{j-3}+3 \Delta t\left({ }^{k} \boldsymbol{F}^{j}+2 \boldsymbol{F}^{j-1}-\boldsymbol{F}^{j-2}\right)\right]$

where superscripts $j$ and $k$ refer to the $j$ th time step and $k$ th inner iteration step, respectively. Inner iterations are required

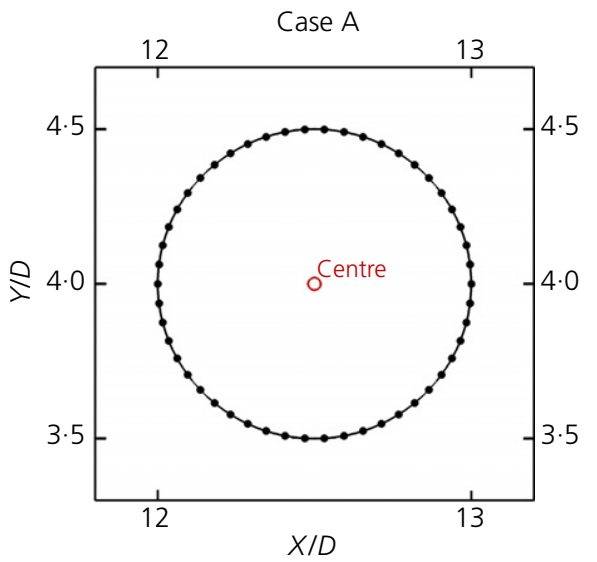

Figure 2. Distribution of Lagrangian forcing points on a circular cylinder. Left: case A, interface forcing; right: case B, solid-domain forcing due to the non-linearity of the coupled system and convergence of the inner iteration is achieved when $\left|{ }^{k+1} q^{j}-{ }^{k} q^{j}\right|<10^{-8}$ (Yang and Balaras, 2006). The truncation error and the final solution of the current time step are calculated as

16. $e^{j}=\frac{9}{121}\left({ }^{k+1} q^{j}-{ }^{p} q^{j}\right)$

and

17. $q^{j}={ }^{k+1} q^{j}-e^{j}$

Figure 1 presents the flowchart of the strongly coupled FSI algorithm.

\section{Example calculations}

While Uhlmann's (2005a) original method and successive similar methods; for example, Kempe and Fröhlich (2012), and Yang et al. (2009) used Lagrangian forcing points only on the immersed boundary itself, the method described above also places Lagrangian points inside the immersed boundary (Uhlmann, 2005b). In the following, several test case flows are simulated using both types of Lagrangian-point placement allowing a direct assessment of validity and accuracy of the two forcing point strategies. Case A refers to simulations for which Lagrangian forcing points are placed only on the immersed boundary (referred to as interface forcing) and simulations of case $\mathrm{B}$ use forcing points on the interface and inside the immersed body (referred to as solid-domain forcing). The distribution of Lagrangian points for the two cases is shown in Figure 2.

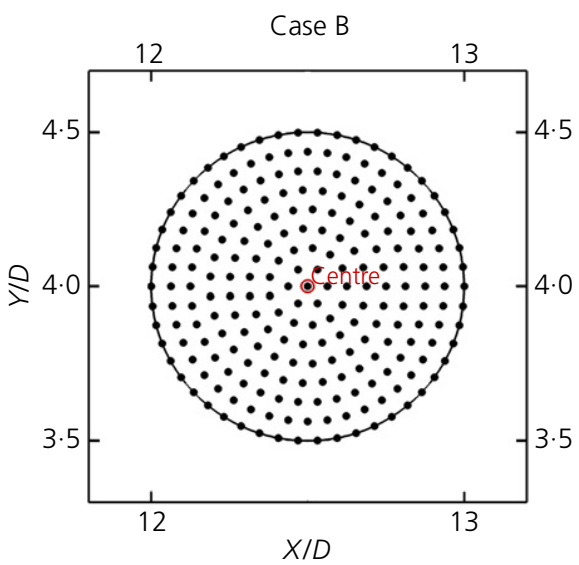




\subsection{Flow past a stationary circular cylinder}

The first test case presented here is the flow past a stationary circular cylinder. Predicting the flow past and forces on a circular cylinder in a free stream is done often for initial FSI code validation due to the availability of data from numerous numerical and experimental studies. In this study, the flow past a circular cylinder at different $\mathrm{Re}$; that is, $\operatorname{Re}=80-185$, is simulated. The computational domain spans $L=52 \mathrm{D}$ and $W=36 D$ in streamwise and spanwise directions, respectively, and uses a total of 900000 grid points. The grid spacing around the immersed interface is $h=0 \cdot 0156 D$. A uniform velocity distribution and a convective boundary condition are applied at inlet and outlet boundaries. At the lateral field boundaries, a slip condition is used.

The reliability of the method in correctly predicting the shedding of vortices is first confirmed by comparing computed Strouhal numbers with data from experiments and similar computational studies. The Strouhal number as a function of Reynolds number of the two cases is plotted in Figure 3, together with data from experimental and numerical studies (Norberg, 2003; Shu et al., 2007; Williamson, 1988b, 1989). Both forcing methods yield very good agreement with the validation data; interface forcing and solid-domain forcing produce virtually identical results in terms of the Strouhal number. Table 1 serves to compare quantitatively the simulated integral properties $c_{\mathrm{D}}, c_{\mathrm{L}}$ and the Strouhal number with experimental and numerical data. Again, the Strouhal number predictions appear to be insensitive to the forcing point placement strategy; however, the drag coefficient predictions are quite different for different methods. Interface forcing methods appear to suffer a parasitic flow inside the immersed boundary (e.g. Kempe and Fröhlich, 2012) and non-smooth pressure distributions across the immersed boundary (see Figure 4), resulting in drag coefficient overestimation, while other methods (e.g. Shu et al. (2007), and Yang and Balaras (2006), and the present solid-domain forcing method) predict lower values of $c_{\mathrm{D}}$.

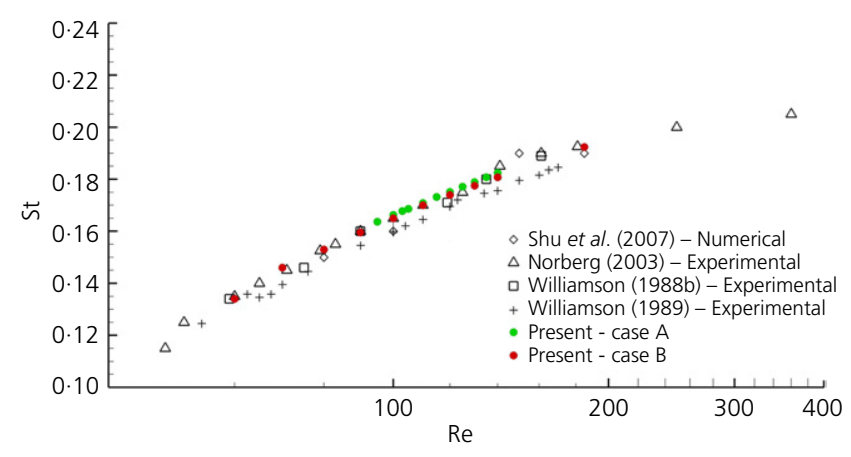

Figure 3. Calculated Strouhal number, St, as a function of the Reynolds number, Re, for flow around a stationary circular cylinder

\begin{tabular}{llll} 
Authors & $C_{D}$ & $C_{L}$ & St \\
\hline Lai and Peskin (2000) & 1.447 & 0.330 & $\mathrm{n} / \mathrm{a}$ \\
Kim et al. (2001) & 1.330 & 0.320 & $\mathrm{n} / \mathrm{a}$ \\
Tseng and Ferziger (2003) & 1.420 & 0.290 & 0.164 \\
Uhlmann (2005a) & 1.453 & 0.339 & 0.169 \\
Shu et al. (2007) & 1.383 & 0.350 & $\mathrm{n} / \mathrm{a}$ \\
Yang et al. (2008) & 1.393 & 0.335 & 0.165 \\
Case A & 1.431 & 0.310 & 0.166 \\
Case B & 1.367 & 0.338 & 0.165
\end{tabular}

Table 1. Calculated time-averaged lift and drag coefficients and Strouhal number

Interestingly, lift coefficients are influenced only marginally by the method of forcing.

\subsection{Prescribed in-line oscillation of a circular cylinder in a fluid at rest}

The efficiency, suitability and accuracy of this method for force predictions in moving boundary problems are evaluated through simulations of the flow past an in-line-oscillating cylinder, for which experimental and numerical data are available for comparison. The two characteristic parameters for an oscillating structure in a fluid at rest are the cylinder Reynolds number, Re, and the Keulegan-Carpenter number, $\mathrm{KC}=\boldsymbol{V}_{\max } / f D$, in which $\boldsymbol{V}_{\max }$ is the maximum velocity of the cylinder and $f$ is the characteristic frequency of oscillation. A simple harmonic in-line oscillatory motion of the cylinder is prescribed as $x(t)=-A \sin (2 / \pi f t)$ where $x(t)$ is the position of the cylinder centre in the direction of oscillation and $A$ is the amplitude of oscillation. The parameters chosen correspond to the experiments and numerical studies reported by Dutsch et al. (1998) for which $\mathrm{Re}=100$ and $\mathrm{KC}=5$. For this set-up the flow remains $2 \mathrm{D}$ with periodic vortex shedding. The same domain size; that is, $52 D \times 36 D$, is used as in the stationary cylinder simulations and von Neumann boundary conditions for velocity and pressure are applied at all far-field boundaries. All computations are started from a zero-velocity flow field and once periodic vortex shedding is obtained, velocities and forces are collected and processed.

Figure 5 quantifies the computed time evolution of the in-line drag force, $F_{x}$, acting on the cylinder in comparison with data from the boundary-conforming, high-resolution simulation of Dutsch et al. (1998). The agreement of the present simulation with the reference computation is very good in terms of phase position and magnitude suggesting accurate prediction of acting forces on moving structures. Noteworthy is the fact that the predicted force as a function of time curve is very smooth; that is, the numerical method does not suffer from numerical 

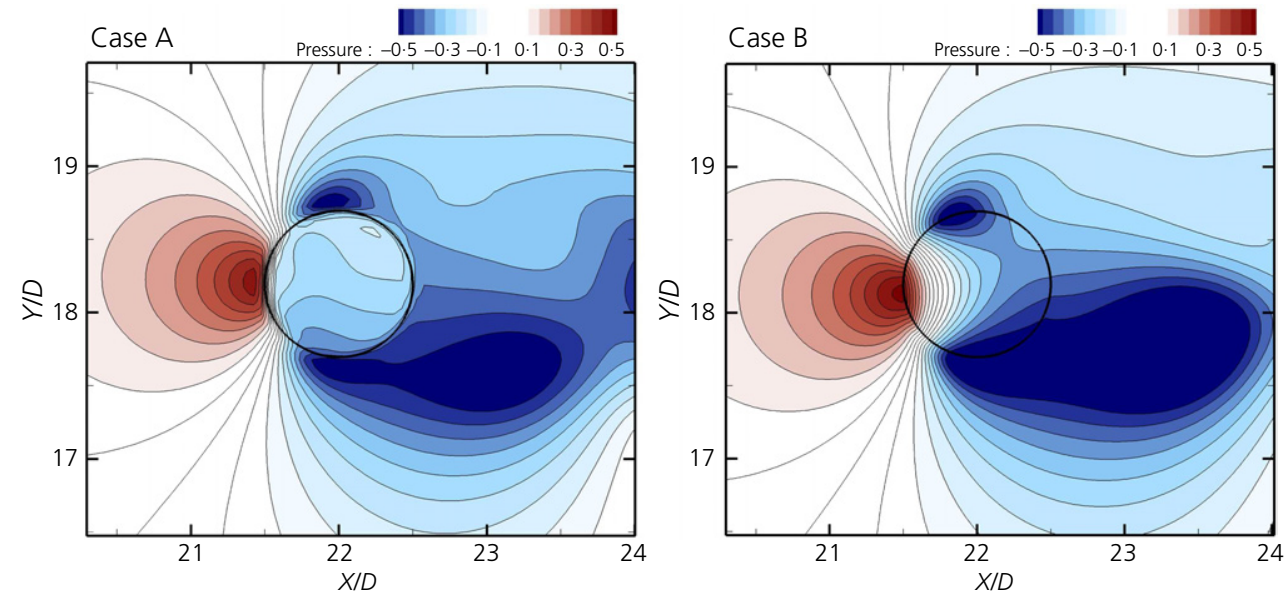

Figure 4. Pressure contours around a cylinder oscillating in the transverse direction: case A (left) and case B (right)

oscillations as, for instance, reported by Seo and Mittal (2011), who attribute these numerical oscillations to mass defects resulting from freshly cleared cells as the cylinders move through the Eulerian grid. As will be shown below, employment of Uhlmann's method together with an adequate choice of regularised delta function remedies the occurrence of numerical oscillations. Further validation of the accuracy of the numerical method is performed by comparing simulated velocity profiles with measured velocity profiles. Dutsch et al. (1998) used a velocity Doppler anemometry system to obtain velocity data at selected locations and during several phases of cylinder displacement. Figure 6 presents measured and computed velocity profiles in the axial (in the direction of movement, left) and in the transverse (right) directions at four different axial locations at a phase angle of $180^{\circ}$; that is, when the cylinder crosses the centre of oscillation. The velocity profiles of the present simulation show fairly good agreement with experimental data for both velocity components and the results are very similar to those obtained by other numerical studies

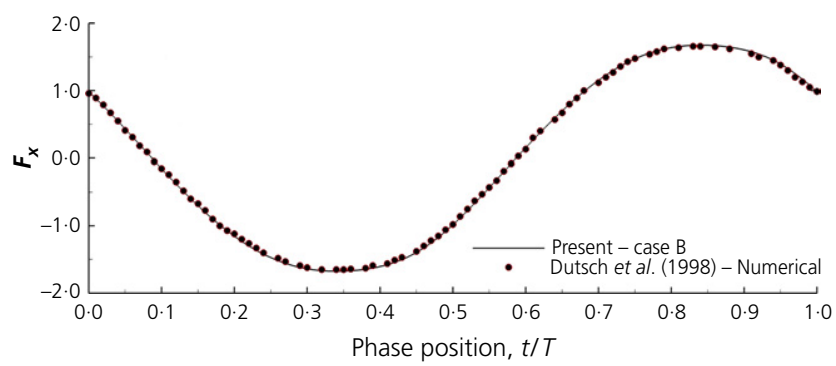

Figure 5. Drag force on a cylinder oscillating in a quiescent fluid at $\mathrm{Re}=100$ and $\mathrm{KC}=5$ as a function of phase position
(Choi et al., 2007; Dutsch et al., 1998; Yang and Balaras, 2006).

\subsection{Prescribed transversely oscillating circular cylinder in a free stream}

A transversely oscillating cylinder in a cross-flow is simulated, a flow problem resembling VIV and a popular test case for FSI numerical model validation. The Reynolds number based on the diameter of the cylinder is set to $\operatorname{Re}=185$. The cylinder is forced to displace in a sinusoidal manner in transverse direction to the flow with an amplitude of $A=0 \cdot 2 D$ and a frequency of $f=0 \cdot 8 f_{\mathrm{s}}$ in which $f_{\mathrm{s}}$ is the natural frequency of the stationary cylinder at $\operatorname{Re}=185$. The structural motion of the cylinder with $f=0 \cdot 156 U / D$ is prescribed as $y(t)=-A \sin (2 / \pi f t)$, identical to what was utilised in Uhlmann (2005a) and Yang et al. (2009). The same domain size and boundary conditions as in the stationary cylinder simulations are used. The time step is selected to be $\Delta t=3 \times 10-3 D / U$, which ensured $\mathrm{CFL}_{\max } \approx 0 \cdot 2$. The results of the computations together with other researcher's data are summarised in Table 2. The current simulations are comparable with other studies in terms of predicted integral properties. The interface forcing method leads to a slightly higher drag coefficient than the solid-domain forcing and the former is comparable with the one reported in Uhlmann (2005a). The solid-domain method's predictions of the drag coefficient corresponds very well with the one obtained in Yang et al. (2009), also in terms of the root mean square (rms) values of the drag and lift force coefficients. Guilmineau and Queutey (2002), who used a body-fitted approach, predicted a somewhat lower drag coefficient than all other methods.

In Figure 7, time variations of the lift and drag coefficients as a function of cylinder displacement are plotted for interface 
Calculation of fluid-structure interaction:

methods, refinements, applications

Kara, Stoesser and McSherry

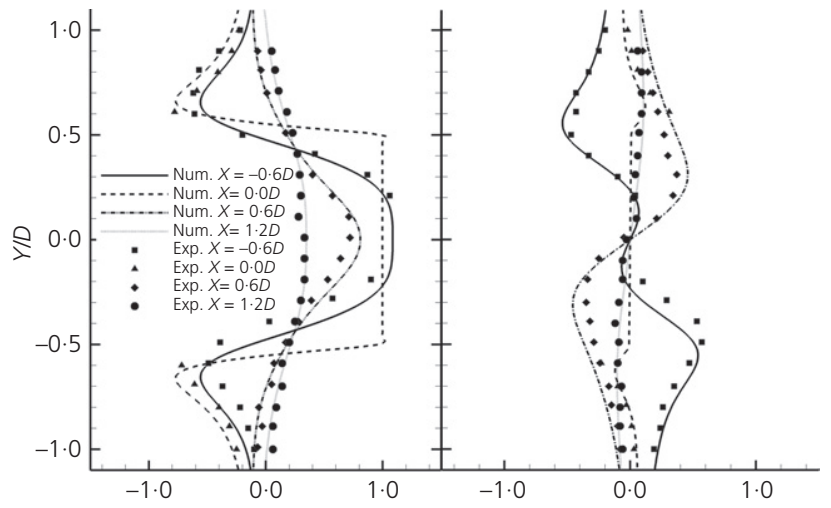

Figure 6. In-line oscillating cylinder in a quiescent fluid at $R e=100$ and $K C=5$. Velocity profiles at a phase angle of $180^{\circ}$. Lines are the present computation; symbols are the experimental data of Dutsch et al. (1998) at $x=-0.6 D, 0.0 D, 0.6 D$ and $1.2 D$

forcing (case A, left) and solid-domain forcing (case B, right). The solid line represents simulation results when using the smooth Dirac delta function of Yang et al. (2009) and the dashed line when using the Dirac delta function as proposed in Uhlmann (2005a). As expected, when using a smooth Dirac delta function, all distributions are smooth and hardly any noticeable numerical oscillations are observed. As shown above for the single stationary cylinder cases, case A (interface forcing) exhibits higher values of the drag force as case B, which results in an upshift of the infinity-sign shape that describes the drag coefficient as a function of cylinder displacement. The values of $c_{\mathrm{D}}$ for case $\mathrm{A}$ are comparable with the ones reported in Uhlmann (2005a). The lift coefficient distribution as a function of displacement is characterised as a 'skewed-zero' shape. The effect of solid-domain forcing on the lift force is quite small and overall slightly higher values of $c_{\mathrm{L}}$ are observed for case B, than for case A. The lift coefficients predicted by the interface forcing method are almost identical in magnitude as the ones reported in Uhlmann (2005a), but a bit smoother due to the use of a smooth Dirac delta function.

\begin{tabular}{llll}
\hline Authors & $C_{\mathrm{D}}$ & $c_{\mathrm{D}}^{\prime}$ & $c_{\mathrm{L}}^{\prime}$ \\
\hline Uhlmann (2005a) & 1.354 & $\mathrm{n} / \mathrm{a}$ & 0.166 \\
Yang et al. (2008) & 1.276 & 0.043 & 0.073 \\
Guilmineau and Queutey (2002) & 1.195 & 0.036 & 0.080 \\
Case A & 1.389 & 0.049 & 0.055 \\
Case B & 1.273 & 0.043 & 0.072
\end{tabular}

Table 2. Calculated time-averaged drag coefficient $C_{D}$ and fluctuation of the drag $c_{\mathrm{D}}^{\prime}$ and lift $c_{\mathrm{L}}^{\prime}$ coefficients, of a transversely oscillating circular cylinder at $\operatorname{Re} D=185$
Figure 4 depicts pressure contours around the oscillating cylinder at arbitrary instants of time. For both forcing methods, the pressure contours around the structure are fairly smooth. However, the distribution of the pressure across the cylinder boundary in case A; that is, interface forcing, is less smooth and steep gradients occur between the outside and inside of the structure. Solid-domain forcing, case B, yields a much smoother pressure field across the cylinder boundary, contour lines appear to be orthogonal to the boundary and a zero-gradient von Neumann-type pressure boundary condition is maintained. This leads to a continuous pressure across the interface and thus results in a sharper resolution of the boundary in terms of velocity distribution (not shown for brevity).

\subsection{VIV of a circular cylinder in the transverse direction}

Vortex shedding of flow over (or around) bluff structures can cause vibrations if the structure's stiffness is not infinitely large. Such VIV are of concern especially in a condition of resonance, which occurs when the vortex-shedding frequency 'locks-in' with the structure's natural frequency, resulting in large amplitudes of cross-stream excitation of the structure. A most relevant test case for the study of VIV is a circular cylinder undergoing transverse oscillations due to vortex shedding. The system is being modelled as a mass-damper-spring system. The simulations are in accordance with the experiment of Anagnostopoulos and Bearman (1992), whose data are used to assess the accuracy and reliability of the simulations. The experiments covered cylinder Reynolds numbers between $\mathrm{Re}=90$ and 140 with reduced velocities ranging from $\boldsymbol{U}_{\text {red }}=5.02$ to $7 \cdot 81$. In addition to the experimental data, numerical simulation results (Kara et al., 2012b; Li et al., 2002; Nomura, 1993; Schulz and Kallinderis, 1998; Wei et al., 1995; Yang et al., 2008) are available for model comparison. Figure 8 shows a schematic representation of a cylinder that is elastically mounted and is allowed to move in the transverse direction. The simulation featured a mass ratio of $m^{*}=150$ and a damping ratio of $\beta=0 \cdot 0012$, chosen to match those of the experiment. The numerical grid and the boundary conditions are exactly the same as in the stationary and prescribed motion cylinder simulations. The flow around stationary cylinders at $\operatorname{Re}=90,95, \ldots, 135,140$ (i.e. 11 simulations) is simulated first to develop quasi-steady vortex shedding. Then, the cylinder is allowed to move in the cross-stream direction until a steady-state phase; that is, when the cylinder has reached a constant maximum displacement amplitude.

The large mass ratio used in these numerical simulations makes the problem fairly stiff and very long integration times are needed for most cases, especially where resonance occurs. Figure 9 presents time histories of cylinder displacement in the 

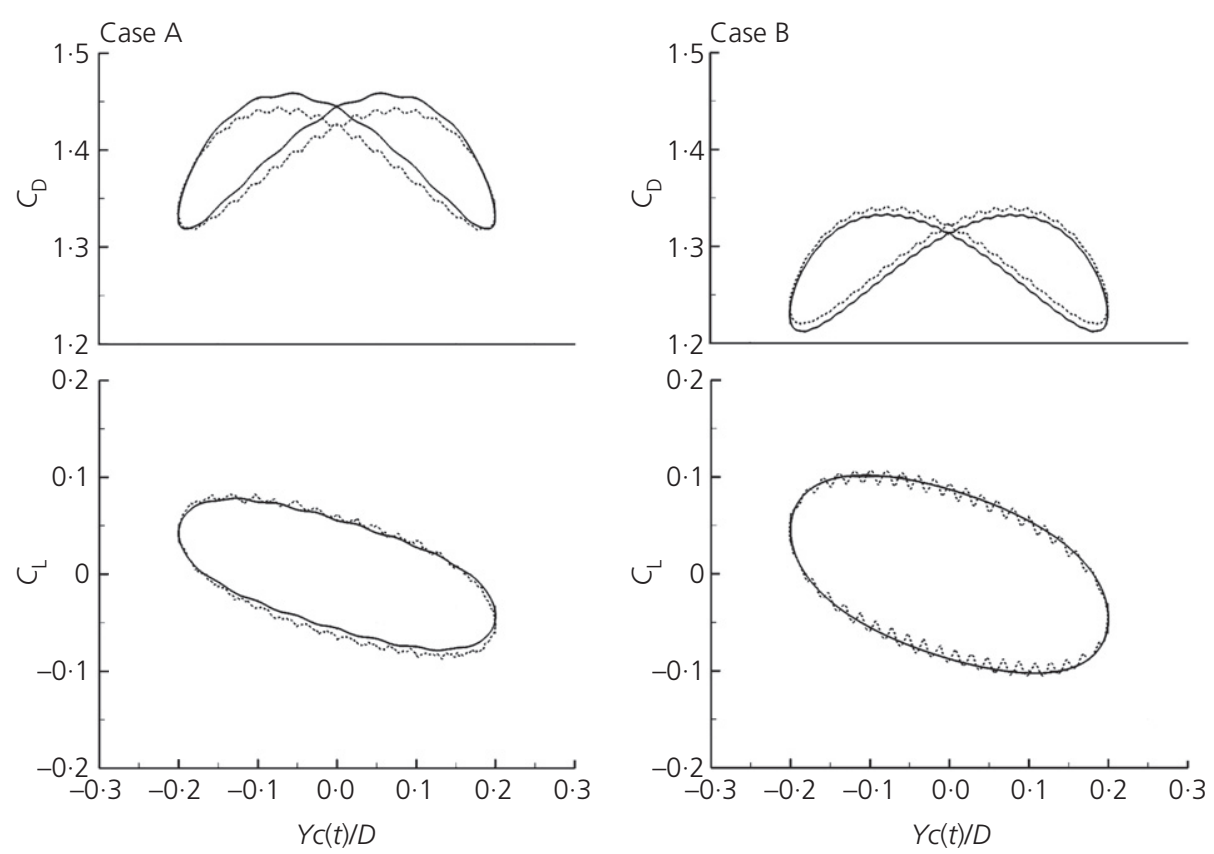

Figure 7. Time variations of the drag (upper row) and lift (lower row) coefficients as a function of cylinder displacement for case $A$ (left) and case B (right)

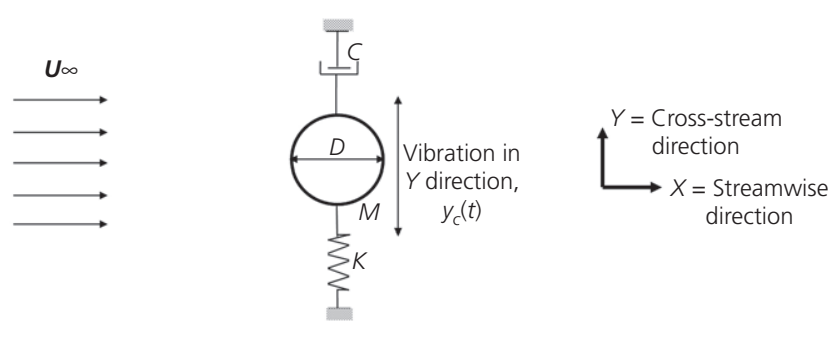

Figure 8. Schematic diagram of a mass-spring-damper system for the simulation of VIV by a flow around an elastically mounted circular cylinder

transverse direction for $\mathrm{Re}=95,105$ and 115 for the start-up phase (upper image of each pair) and the steady-state phase (lower image of each pair). Outside the resonance regime, such as at $R e=95$, the amplitude of vibration varies periodically and the steady state is characterised by a relatively small magnitude of cylinder displacement. A similar behaviour is observed at $\mathrm{Re}=130-140$ (not shown for brevity); however, the displacement history appears to be more irregular than for the cases with $\mathrm{Re}=95$. Oscillations monotonically grow at $\mathrm{Re}=105$ and within the lock-in regime (steady state), the displacement amplitude increases to approximately ten times its value as compared with outside the lock-in regime.
A quantitative evaluation of the predicted FSI is provided with the help of Figures 10 and 11, which present the maximum oscillation amplitude, $A_{\max }$, as a function of Re (Figure 10) and the frequency ratio between vortex shedding $(f)$ and natural frequency $\left(f_{\mathrm{N}}\right), f^{*}=f l f_{\mathrm{N}}$, as a function of reduced velocity, $\boldsymbol{U}_{\text {red }}$ (Figure 11). Also plotted in these figures are data from previous numerical simulations (Li et al., 2002; Schulz and Kallinderis, 1998; Wei et al., 1995; Yang et al., 2008) and the experimentally predicted behaviour (Anagnostopoulos and Bearman, 1992). All previous computations use boundaryfitted methods, except Yang et al. (2008), whose method is quite similar to the one presented here. All computations predict the amplitude of oscillation very well, as well as the extent of the lock-in regime. However, the present simulation, regardless of forcing point treatment, suggests the onset of the lock-in regime to be at $\operatorname{Re} \approx 100$, which is fairly close to the experimentally obtained value of $\operatorname{Re}=103$, while the other simulations suggest this to occur a bit earlier; that is, at $\operatorname{Re} \approx 95$. However, the present method predicts that at $\mathrm{Re}=115$ FSI is not locked-in anymore, although experiments suggest the lock-in regime to extend until $\mathrm{Re} \approx 130$. Similar findings are reported in other computations and discrepancies are attributed to $3 \mathrm{D}$ effects of the experimental set-up, which $2 \mathrm{D}$ simulations cannot represent. Noteworthy is the fact that the soliddomain forcing provides slightly larger oscillation amplitudes. The computed normalised oscillation frequency as plotted in 

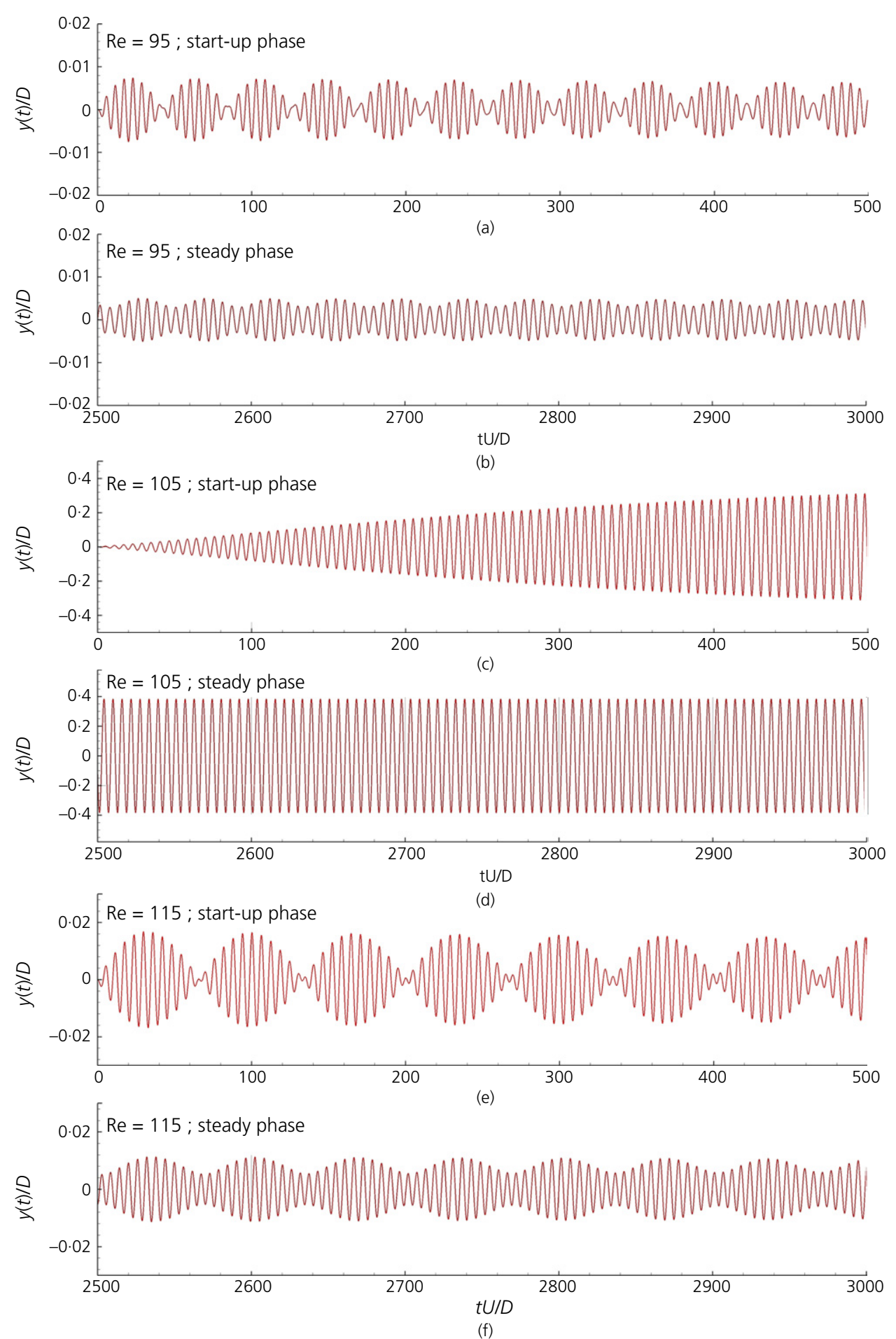

Figure 9. Time history of cylinder displacement in the transverse direction for ( $a$ and b) $\operatorname{Re}=95$, ( $c$ and d) $\operatorname{Re}=105$ and (e and $f$ ) $R e=115$. The upper image of each pair depicts the start-up phase and the lower image of the figure depicts the steady phase 


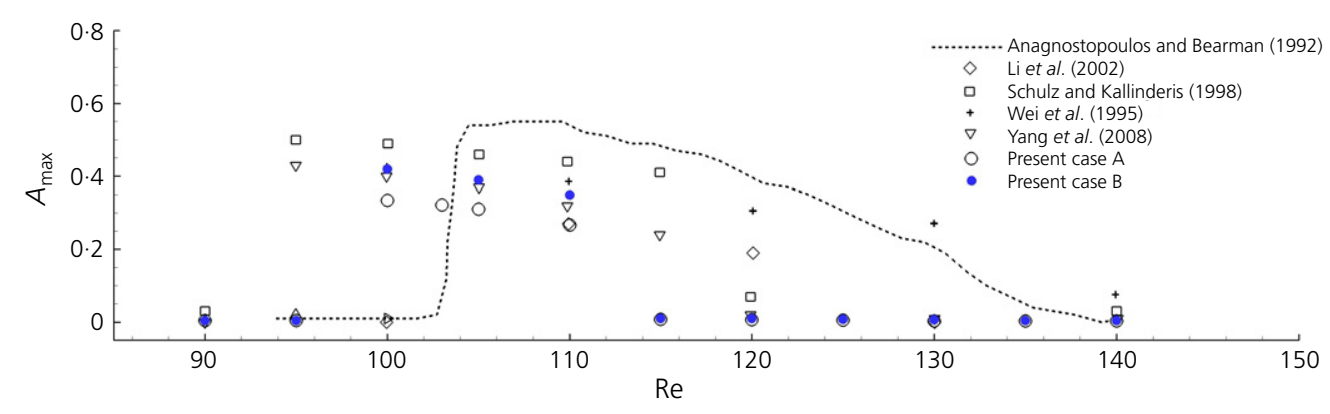

Figure 10. Maximum oscillation amplitude $A_{\max }$ as a function of

$\operatorname{Re} D$

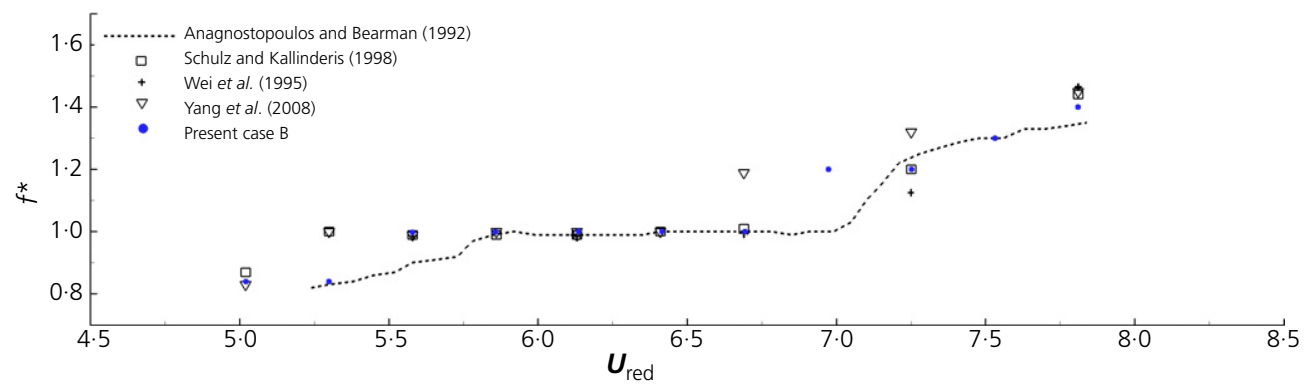

Figure 11. Frequency ratio between vortex shedding $(f)$ and

natural frequency $\left(f_{N}\right), f^{\star}=f / f_{N}$, as a function of reduced velocity,

$\boldsymbol{U}_{\text {red }}$

Figure 11 compares quite well with the experimentally determined one, again onset and offset of the lock-in is a bit shifted towards lower reduced velocities; nevertheless, the physical behaviour of this complex FSI problem is reproduced fairly accurately.

\section{Conclusions}

We have presented a brief review of the most significant previous studies on flows past stationary and moving circular cylinders, including those undergoing fluid dynamic excitation in the form of VIV. The review highlights the very significant contributions to our present understanding of this classic flow problem that have been made possible by the vast number of numerical studies that have been undertaken since the 1970s. Particular contributions have been made regarding transition to $3 \mathrm{D}$ vortex shedding, the branching behaviour of cylinders with low mass and damping in VIV and spanwise vortex dynamics in long slender cylinders. The review also highlights the importance of flow past a circular cylinder as a convenient validation test case for new numerical methods, with particular focus on the IBM. Variations on the IBM, be they direct forcing methods, sharp-interface methods or hybrids of the two, have consistently been proven to be capable of capturing accurately and efficiently the key features of flows past circular cylinders, providing good agreement with experimental data in terms of force coefficients and Strouhal number. Furthermore, VIV responses and lock-in behaviour have also been well predicted by the IBM for low Reynolds number test cases.

The authors have also introduced and applied an immersed boundary-method-based Cartesian grid FSI solver to predict accurately FSI and VIV. The IBM is a refined version of previous methods and uses improved smoothing techniques and in addition to the common interface forcing a solid-domain forcing strategy. The fluid solver is strongly coupled with the equation of motion through Hamming's fourth-order predictor-corrector method, which allows computation of the interaction of a fluid with a moving structure. The accuracy, validity and efficiency of the proposed method have been demonstrated by a series of validation cases including flow past stationary and moving cylinders, both prescribed and freely vibrating, for which laboratory and numerical simulation data are available. The comparisons of the results obtained using both interface and solid-domain forcing strategies with 
Engineering and Computational Mechanics Volume 168 Issue EM2
Calculation of fluid-structure interaction: methods, refinements, applications

Kara, Stoesser and McSherry available experimental and numerical data have shown that the solid-domain forcing approach is able to deliver quite accurate numerical predictions of both drag and lift forces on the immersed structure for fixed and moving boundaries. The advantage of solid-domain forcing is the absence of the parasitic flow in the interior of the immersed boundary, which improves numerical predictions of drag forces. The numerical method reproduces fairly well the behaviour of an elastically mounted cylinder subjected to a fluid flow. In particular, transverse oscillation amplitude as well as oscillation frequency are computed quite accurately. In addition, the predicted vortexinduced cylinder vibration lock-in regime is in good agreement with the laboratory experiment.

\section{Acknowledgements}

Some of the computations presented in this paper were carried out on Cardiff University's Supercomputer Raven, hosted and maintained by Advanced Research Computing @ Cardiff (ARCCA). Provision of computing time and support is gratefully acknowledged.

\section{REFERENCES}

Al-Jamal H and Dalton C (2004) Vortex induced vibrations using large eddy simulation at moderate Reynolds number.

Journal of Fluids and Structures 19(1): 73-92.

Anagnostopoulos P and Bearman PW (1992) Response characteristics of a vortex-excited cylinder at low Reynolds numbers. Journal of Fluids and Structures 6(1): 39-50.

Barkley R and Henderson R (1996) Three-dimensional Floquet stability analysis of the wake of a circular cylinder. Journal of Fluid Mechanics 322: 215-241.

Beaudan P and Moin P (1994) Numerical Experiments on the Flow Past a Circular Cylinder at Sub-Critical Reynolds Number. Department of Mechanical Engineering, Stanford University, Stanford, CA, USA, Report TF-62.

Blackburn HM, Govardhan RN and Williamson CHK (2001) A complementary numerical and physical investigation of vortex-induced vibration. Journal of Fluids and Structures 15(3-4): 481-488.

Blevins RD (1990) Flow-Induced Vibrations. Van Nostrand Reinhold, New York, NY, USA

Bomminayuni S and Stoesser T (2009) Turbulence statistics in an open-channel flow over a rough bed. Journal of Hydraulic Engineering 137(11): 1347-1358.

Borazjani I and Sotiropoulos F (2009) Vortex-induced vibrations of two cylinders in tandem arrangement in the proximitywake region. Journal of Fluid Mechanics 621: 321-364.

Breuer M (1998) Numerical and modeling influences on large eddy simulations for the flow past a circular cylinder. International Journal of Heat and Fluid Flow 19(5): 512-521.

Breuer M (2000) A challenging test case for large eddy simulation: high Reynolds number circular cylinder flow.
International Journal of Heat and Fluid Flow 21(5): 648-654.

Bunner B (1998) MG NET. See http://www.mgnet.org/mgnetcodes-bunner.html (accessed 16/04/2015).

Cantwell B and Coles D (1983) An experimental study on entrainment and transport in the turbulent near wake of a circular cylinder. Journal of Fluid Mechanics 136: 321-374.

Carnahan B, Luther HA and Wilkes JO (1969) Applied Numerical Methods. John Wiley, New York, NY, USA.

Chaplin JR (2005) Blind predictions of laboratory measurements of vortex-induced vibrations of a tension riser. Journal of Fluids and Structures 21(1): 25-40.

Choi Jl, Oberoi RC, Edwards JR and Rosati JA (2007) An immersed boundary method for complex incompressible flows. Journal of Computational Physics 224(2): 757-784.

Dong S and Karniadakis GE (2005) DNS of flow past a stationary and oscillating cylinder at $\mathrm{Re}=10000$. Journal of Fluids and Structures 20(4): 519-531.

Dong S, Karniadakis GE, Ekmekci A and Rockwell D (2006) A combined direct numerical simulation-particle image velocimetry study of the turbulent near wake. Journal of Fluid Mechanics 569: 185-207.

Dutsch H, Durst F, Becker S and Lienhart H (1998) LowReynolds-number flow around an oscillating circular cylinder at low Keulegan-Carpenter numbers. Journal of Fluid Mechanics 360: 249-271.

Fadlun EA, Verzicco R, Orlandi P and Mohd-Yusof J (2000) Combined immersed-boundary finite-difference methods for three-dimensional complex flow simulations. Journal of Computational Physics 161(1): 35-60.

Feng CC (1968) The Measurement of Vortex-Induced Effects in Flow Past Stationary and Oscillating Circular and D-Section Cylinders. Masters thesis, University of British Columbia, Vancouver, Canada.

Gazzola M, Chatelain P, van Rees WM and Koumoutsakos P (2011) Simulations of single and multiple swimmers with non-divergence free deforming geometries. Journal of Computational Physics 230(19): 7093-7114.

Germano U, Piomelli P, Moin P and Cabot WH (1991) A dynamic subgrid scale eddy viscosity model. Physics of Fluids A 3(7): 1760-1765.

Gilmanov A and Sotiropoulos F (2005) A hybrid Cartesian/ immersed boundary method for simulating flows with 3D, geometrically complex, moving bodies. Journal of Computational Physics 207(2): 457-492.

Gopalkrishnan R (1993) Vortex-Induced Forces on Oscillating Bluff Cylinders. $\mathrm{PhD}$ thesis, Massachusetts Institute of Technology, Cambridge, MA, USA.

Govardhan R and Williamson CHK (2000) Modes of vortex formation and frequency response of a freely vibrating cylinder. Journal of Fluid Mechanics 420: 85-130. 
Govardhan R and Williamson CHK (2006) Defining the 'modified Griffin plot' in vortex-induced vibration: revealing the effect of Reynolds number using controlled damping. Journal of Fluid Mechanics 561: $147-180$.

Griffin OM (1980) Vortex-excited cross-flow vibrations of a single cylindrical tube. ASME Journal of Pressure Vessel Technology 102(2): 158-166.

Guilmineau E and Queutey P (2002) A numerical simulation of vortex shedding from an oscillating circular cylinder. Journal of Fluids and Structures 16(6): 773-794.

Henderson RD (1997) Nonlinear dynamics and pattern formation in turbulent wake transition. Journal of Fluid Mechanics 352: 65-112.

Henderson RD and Karniadakis GE (1995) Unstructured spectral element methods for simulation of turbulent flows. Journal of Computational Physics 122(2): 191-217.

Hover FS, Davis JT and Triantafyllou MS (2004) Threedimensionality of mode transition in vortex-induced vibrations of a circular cylinder. European Journal of Mechanics - B/Fluids 23(1): 29-40.

laccarino $\mathrm{G}$ and Verzicco R (2003) Immersed boundary technique for turbulent flow simulations. Applied Mechanics Reviews 56(3): 331-347.

Kara MC, Stoesser T and Will KM (2012a) A numerical method to predict fluid-structure interaction of flow past an elastically mounted circular cylinder. Proceedings of 22nd International Offshore and Polar Engineering Conference, ISOPE, Rhodes, Greece.

Kara S, Stoesser T and Sturm TW (2012b) Turbulence statistics in compound channels with deep and shallow overbank flows. Journal of Hydraulic Research 50(5): 482-493.

Kempe T and Fröhlich J (2012) An improved immersed boundary method with direct forcing for the simulation of particle laden flows. Journal of Computational Physics 231(9): 3663-3684.

Khalak A and Williamson CHK (1999) Motions, forces and mode transitions in vortex-induced vibrations at low mass-damping. Journal of Fluids and Structures 13(7-8): 813-851.

Kim D and Choi H (2006) Immersed boundary method for flow around an arbitrarily moving body. Journal of Computational Physics 212(2): 662-680.

Kim D, Kim JH and Stoesser T (2013) The effect of baffle spacing on hydrodynamics and solute transport in serpentine contact tanks. Journal of Hydraulic Research 51(5): 558-568.

Kim J, Kim D and Choi H (2001) An immersed-boundary finite-volume method for simulations of flow in complex geometries. Journal of Computational Physics 171(1): 132-171.

Kim SJ and Stoesser T (2011) Closure modeling and direct simulation of vegetation drag in flow through emergent vegetation. Water Resources Research 47(10): W10511.

Kolomenskiy D and Scheider K (2009) A Fourier spectral method for the Navier-Stokes equations with volume penalization for moving solid obstacles. Journal of Computational Physics 228(16): 5687-5709.

Kravchenko AG and Moin P (1998) B-Spline Methods and Zonal Grids for Numerical Simulations of Turbulent Flows. Department of Mechanical Engineering, Stanford University, Stanford, CA, USA, Report TF-73.

Lai MC and Peskin CS (2000) An immersed boundary method with formal second-order accuracy and reduced numerical viscosity. Journal of Computational Physics 160(2): 705-719.

Leontini JS, Thompson MC and Hourigan K (2006) The beginning of branching of vortex-induced vibration during two-dimensional flow. Journal of Fluids and Structures 22(6-7): 857-864.

Li L, Sherwin SJ and Bearman PW (2002) A moving frame of reference algorithm for fluid/structure interaction of rotating and translating bodies. International Journal for Numerical Methods in Fluids 38(2): 187-206.

Lighthill J (1986) Fundamentals concerning wave loading on offshore structures. Journal of Fluid Mechanics 173: 667-681.

Lu XY and Dalton C (1996) Calculation of the timing of vortex formation from an oscillating cylinder. Journal of Fluids and Structures 10(5): 527-541.

Meneghini JR and Bearman PW (1995) Numerical simulation of high amplitude oscillatory flow about a circular cylinder. Journal of Fluids and Structures 9(4): 435-455.

Mittal R and Moin P (1997) Suitability of upwind-biased finite-difference schemes for large-eddy simulation of turbulent flows. AIAA Journal 35(8): 1415-1417.

Morse TL and Williamson CHK (2009) Prediction of vortex-induced vibration response by employing controlled motion. Journal of Fluid Mechanics 634: 5-39.

Newman D and Karniadakis GE (1996) Simulations of flow over a flexible cable: a comparison of forced and flow-induced vibration. Journal of Fluids and Structures 10(5): 439-453.

Newman D and Karniadakis GE (1997) A direct numerical simulation study of flow past a freely vibrating cable. Journal of Fluid Mechanics 344: 95-136.

Nomura T (1993) Finite-element analysis of vortex-induced vibrations of bluff cylinders. Journal of Wind Engineering and Industrial Aerodynamics 46-7: 587-594.

Norberg C (2003) Fluctuating lift on a circular cylinder: review and new measurements. Journal of Fluids and Structures 17(1): 57-96.

Ongoren A and Rockwell D (1988) Flow structure from an oscillating cylinder. Part 1: mechanisms of phase shift and 
recovery in the near wake. Journal of Fluid Mechanics 191: 197-223.

Papanicolaou AN, Kramer CM, Tsakiris AG et al. (2012) Effects of a fully submerged boulder within a boulder array on the mean and turbulent flow fields: implications to bedload transport. Acta Geophysica 60(6): 1502-1546.

Peskin CS (1972) Flow patterns around heart valves numerical method. Journal of Computational Physics 10(2): 252-271.

Peskin CS (1977) Numerical-analysis of blood-flow in heart. Journal of Computational Physics 25(3): 220-252.

Peskin CS (2002) The immersed boundary method. Acta Numerica 11: 479-517.

Preidikman S and Mook D (2000) Time-domain simulations of linear and nonlinear aeroelastic behavior. Journal of Vibration and Control 6: 1135-1175.

Rodi W, Constantinescu G and Stoesser T (2013)

Large-Eddy Simulation in Hydraulics. IAHR Monographs. CRC Press, Leiden, the Netherlands.

Roshko A (1954) On the Development of Turbulent Wakes from Vortex Streets. NACA Report 1191, Washington DC, USA.

Sarpkaya T (2004) A critical review of the intrinsic nature of vortex-induced vibrations. Journal of Fluids and Structures 19(4): 389-447.

Schulz KW and Kallinderis Y (1998) Unsteady flow structure interaction for incompressible flows using deformable hybrid grids. Journal of Computational Physics 143(2): 569-597.

Seo JH and Mittal R (2011) A sharp-interface immersed boundary method with improved mass conservation and reduced spurious pressure oscillations. Journal of Computational Physics 230(19): 7347-7363.

Sheard GJ, Thompson MC and Hourigan K (2005) Subharmonic mechanism of the mode C instability. Physics of Fluids 17(11): 111702.

Shiels D, Leonard A and Roshko A (2001) Flow-induced vibration of a circular cylinder at limiting structural parameters. Journal of Fluids and Structures 15(1): 3-21.

Shin SJ, Huang W-X and Sung HJ (2008) Assessment of regularized delta functions and feedback forcing schemes for an immersed boundary method. International Journal for Numerical Methods in Fluids 58(3): 263-286.

Shu C, Liu N and Chew YT (2007) A novel immersed boundary velocity correction-lattice Boltzmann method and its application to simulate flow past a circular cylinder. Journal of Computational Physics 226(2): $1607-1622$.

Singh SP and Mittal S (2005) Vortex-induced oscillations at low Reynolds numbers: hysteresis and vortexshedding modes. Journal of Fluids and Structures 20(8): 1085-1104.
Sotiropoulos F and Yang X (2014) Immersed boundary methods for simulating fluid-structure interaction. Progress in Aerospace Sciences 65: 1-21.

Staubli T (1983) Calculation of the vibration of an elastically mounted cylinder using experimental data from forced oscillation. Journal of Fluids Engineering 105(2): 225-229.

Sumer BM and Fredsoe J (1997) Hydrodynamics around Cylindrical Structures. World Scientific, Singapore, Singapore.

Toebes GH (1969) The unsteady flow and wake near an oscillating cylinder. ASME Journal of Basic Engineering 91(3): 493-502.

Tseng YH and Ferziger JH (2003) A ghost-cell immersed boundary method for flow in complex geometry. Journal of Computational Physics 192(2): 593.

Udaykumar HS, Mittal R, Rampunggoon P and Khanna A (2001) A sharp interface Cartesian grid method for simulating flows with complex moving boundaries. Journal of Computational Physics 174(1): 345-380.

Uhlmann M (2005a) An immersed boundary method with direct forcing for the simulation of particulate flows. Journal of Computational Physics 209(2): 448-476.

Uhlmann M (2005b) An improved fluid-solid coupling method for DNS of particulate flow on a fixed mesh. Proceedings of 11th Workshop on Two-Phase Flow Predictions, Merseburg, Germany.

Wei R, Sekine A and Shimura M (1995) Numerical-analysis of $2 \mathrm{D}$ vortex-induced oscillations of a circular-cylinder. International Journal for Numerical Methods in Fluids 21(10): 993-1005.

Willden RHJ (2006) Numerical simulation of the multiple branch transverse response of a low mass ratio elastically supported circular cylinder. Proceedings of the ASME Pressure Vessels and Piping Division Conference, Vancouver, Canada, pp. 881-890.

Willden RHJ and Graham JM (2006) Three distinct response regimes for the transverse vortex-induced vibration of circular cylinders at low Reynolds numbers. Journal of Fluids and Structures 22(6-7): 885-895.

Williamson CHK (1988a) The existence of two stages in the transition to three-dimensionality of a cylinder wake. Physics of Fluids 31(11): 3165-3168.

Williamson CHK (1988b) Defining a universal and continuous Strouhal-Reynolds number relationship for the laminar vortex shedding of a circular-cylinder. Physics of Fluids 31(10): 2742-2744.

Williamson CHK (1989) Oblique and parallel modes of vortex shedding in the wake of a circular-cylinder at low Reynolds-numbers. Journal of Fluid Mechanics 206: 579-627.

Williamson CHK (1996) Vortex dynamics in the cylinder wake. Annual Review of Fluid Mechanics 28: 477-539. 
Williamson CHK and Govardhan R (2004) Vortex-induced vibrations. Annual Review of Fluid Mechanics 36: 413-455.

Williamson CHK and Govardhan R (2008) A brief review of recent results in vortex-induced vibrations. Journal of Wind Engineering and Industrial Aerodynamics 96(6-7): 713-725.

Williamson CHK and Roshko A (1988) Vortex formation in the wake of an oscillating cylinder. Journal of Fluids and Structures 2(4): 355-381.

Yang J, Preidikman S and Balaras E (2008) A strongly coupled, embedded-boundary method for fluid-structure interactions of elastically mounted rigid bodies. Journal of Fluids and Structures 24(2): 167-182.

Yang JM and Balaras E (2006) An embedded-boundary formulation for large-eddy simulation of turbulent flows interacting with moving boundaries. Journal of Computational Physics 215(1): 12-40.

Yang JM and Stern F (2012) A simple and efficient direct forcing immersed boundary framework for fluid-structure interactions. Journal of Computational Physics 231(15): 5029-5061.

Yang XL, Zhang X, Li ZL and He GW (2009) A smoothing technique for discrete delta functions with application to immersed boundary method in moving boundary simulations. Journal of Computational Physics 228(20): 7821-7836.

Yu X and Shao A (2007) A direct-forcing fictitious domain method for particulate flows. Journal of Computational Physics 227(1): 292-314.

Zdravkovich MM (1997) Flow Around Circular Cylinders, Vol. 1: Fundamentals. Oxford University Press, Oxford, UK.

Zdravkovich MM (2003) Flow Around Circular Cylinders, Vol. 1: Fundamentals. Oxford University Press, Oxford, UK.

Zhang J and Dalton C (1996) Interactions of vortex-induced vibrations of a circular cylinder and a steady approach flow at a Reynolds number of 13000. Computers and Fluids 25(3): 283-294.

\section{WHAT DO YOU THINK?}

To discuss this paper, please email up to 500 words to the editor at journals@ice.org.uk. Your contribution will be forwarded to the author(s) for a reply and, if considered appropriate by the editorial panel, will be published as discussion in a future issue of the journal.

Proceedings journals rely entirely on contributions sent in by civil engineering professionals, academics and students. Papers should be 2000-5000 words long (briefing papers should be 1000-2000 words long), with adequate illustrations and references. You can submit your paper online via www.icevirtuallibrary.com/content/journals, where you will also find detailed author guidelines. 